\title{
Stochastic lot sizing problem with nervousness considerations
}

\author{
E. Koca ${ }^{\mathrm{a}, *}$, H. Yaman ${ }^{\mathrm{b}}$, M.S. Aktürk ${ }^{\mathrm{b}}$ \\ a Industrial Engineering, Sabancı University, Istanbul, Turkey \\ ${ }^{\mathrm{b}}$ Department of Industrial Engineering, Bilkent University, Ankara, Turkey
}

\section{A R T I C L E I N F O}

Article history:

Received 12 October 2016

Revised 26 November 2017

Accepted 30 January 2018

Available online 1 February 2018

\section{Keywords:}

Stochastic lot sizing problem

Controllable processing times

Nervousness

Continuous mixing set

\begin{abstract}
A B S T R A C T
In this paper, we consider the multistage stochastic lot sizing problem with controllable processing times under nervousness considerations. We assume that the processing times can be reduced in return for extra cost (compression cost). We generalize the static and static-dynamic uncertainty strategies to eliminate setup oriented nervousness and control quantity oriented nervousness. We restrict the quantity oriented nervousness by introducing a new concept called promised production amounts, and considering new range constraints and a nervousness cost function. We formulate the problem as a second-order cone mixed integer program (SOCMIP), and apply the conic strengthening. We observe the continuous mixing set substructure in our formulation that arises due the controllable processing times. We reformulate the problem by using an extended formulation for the continuous mixing set and solve the problem by a branch-and-cut approach. The computational experiments indicate that the reformulation reduces the root gaps and this helps to solve the problem in less computation times. Moreover, in our computational experiments we investigate the impact of new restrictions, specifically the additional cost of eliminating the setup oriented nervousness, on the total costs and the system nervousness. Our computational results clearly indicate that we could significantly reduce the nervousness costs and generate more stable production schedules with a relatively small increase in the total cost.
\end{abstract}

(C) 2018 Elsevier Ltd. All rights reserved.

\section{Introduction}

Demand uncertainty is one of the major challenges in stochastic lot sizing problems. There are two extreme strategies used in modeling this problem: static and dynamic uncertainty strategies. In the static uncertainty strategy, the production schedule is decided at the beginning of the planning horizon and implemented without any revision. In the dynamic uncertainty strategy, production decisions are taken dynamically as response to demand realizations. It is possible to find less costly production plans under dynamic uncertainty strategy since more information is obtained until the time of the decision. On the other hand, under this strategy, the production plan is not known in advance, which could cause frequent revisions.

Uncertainty of a production plan or frequent revisions in a production schedule cause a problem in the system which is called "system nervousness" or "planning instability". Nervousness is one of the most important performance measures in the inventory control theory (De Kok and Inderfurth, 1997). The nervousness on the top level of production systems propagates throughout the system

\footnotetext{
* Corresponding author.

E-mail address: ekoca@sabanciuniv.edu (E. Koca).
}

since schedule changes at one level of the production system may lead to adjustments at other levels of the system (De Kok and Inderfurth, 1997). Hence, if the system is not flexible with respect to revisions, nervousness may be a big problem for the whole system, and implementing the revisions may cost more (or may be harder) than implementing a non-optimal production schedule (in terms of cost) that causes less nervousness in the system (Kilic and Tarim, 2011).

Although nervousness is mostly considered as a problem for a production system, nervousness of one system may affect other systems related to that. Besides, nervousness may arise not only in production systems but also in transportation and procurement systems. For example, changes in the ordering plan of a buyer may negatively influence its relation with its supplier (or transporter), since decisions of the buyer may alter the ones of the supplier (or transporter).

In this paper, we introduce a new multistage stochastic lot sizing problem to control the nervousness of the production (or ordering/buying) schedules. We eliminate the setup oriented nervousness, which is caused by changes in the production periods (cancellation of a production decision or deciding to produce in a period that is not considered as a production period before), and reduce quantity oriented nervousness that is related to the 
modifications (increase or decrease) in the decided production amounts by introducing new restrictions.

In the classical scenario tree formulation of the stochastic lot sizing problem, setup and production decisions are taken for each scenario separately. Thus, a decision for a period depends on the demand realization in that period, and hence the resulting solution may lead to both setup and quantity oriented nervousness. In this study, in order to eliminate setup oriented nervousness we take setup decisions for each period, independently from the demand realizations, and to reduce quantity oriented nervousness we restrict the production amounts under different scenarios to be within an interval of promised production (or order) amount for the corresponding period. In other words, the promised production amount for each period is decided in advance, and the actual production amount of a period under any scenario is restricted to be in some certain range of this promised production amount. Since promised production amounts are decided beforehand, one can use these values in arrangements before the production, purchasing or transportation starts. The promised production amounts can be seen as capacity reservations for future periods. For example, a producer can adjust other levels of the system, or a buyer can inform its supplier (or transporter) and reserve capacities for future periods by using these values (see, e.g., van Norden and van de Velde, 2005). Note that, when the promised production amounts are known, one can compute the range that contains all the possible actual production (or order) amounts under different scenarios for each period.

Another way of preventing large deviations from the promised production amount is to penalize the difference between the actual production amounts and the promised production amount. This penalty cost can be motivated from the following example. Consider a buyer-supplier system where the buyer informs the supplier about its promised order amounts for the future periods. The buyer has the flexibility of changing its actual ordering amount within some range of the promised order amounts. On the other hand, the supplier has a right to penalize the difference between the actual and the promised order amounts to protect itself from large deviations. In this case, the buyer has to consider this penalty cost as a part of its total cost function. Note that, by using a convex penalty function the supplier can prevent large deviations from the promised order amounts. In this study, we will consider a convex cost function to penalize the differences between the promised and the actual production amounts under each scenario. We will call this penalty cost the nervousness cost from now on. More specifically, we assume that the nervousness cost is a convex increasing power function in the form of $x^{p / q}$ for $p / q \geq 1$ which is known to be conic quadratic representable (Ben-Tal and Nemirovski, 2001).

In our formulation, we generalize the static and static dynamic uncertainty strategies of Bookbinder and Tan (1988). In the staticdynamic uncertainty strategy, the replenishment periods are determined at the beginning of the planning horizon, and replenishment amounts are decided at the beginning of these periods. Thus, this strategy causes only quantity oriented nervousness. Note that static uncertainty strategy does not cause any nervousness since the production schedule is fixed at the beginning. In our formulation, we include both of these strategies as special cases. To the best of our knowledge, this is the first study that considers the static-dynamic uncertainty strategy in the multistage stochastic lot sizing problem.

In most of the studies on the lot sizing problems, processing times (or capacities) are assumed to be constant. In practice, the processing time of a job can be controlled (and reduced) by changing the machine speed, allocating extra manpower, subcontracting, overloading, consuming additional money or energy, and these options are available in many real life production and inventory systems. In this study, we assume that the processing times can be reduced in return for extra cost (compression cost). For example, increasing a machining speed in a production system will reduce the processing time at the expense of an additional tool consumption cost and may also increase the energy consumption of the facility. On the other hand, by reducing the processing times, or increasing the capacities, one can produce more in a period and consequently, might eliminate the number of production periods. As a result, controllable processing times can be seen as an alternative to holding inventory: instead of producing before and holding inventory, we have an option to produce more in subsequent periods by reducing the processing times.

In a buyer-supplier system, where the supplier has limited capacities, reducing the processing times can be conceived as ordering more than the capacity of the supplier. In this case, the supplier might use subcontracting, outsourcing, or overtime to satisfy the demand of the buyer, but may charge more than the usual cost for the product. Similarly, in a customer-transporter system, if the customer orders more than the capacity of a truck, the transporter might overload trucks (up to some limit), and may charge extra cost due to increased fuel consumption and/or carbon emission.

Overall, we consider a system where processing times can be reduced or capacities can be increased. Besides, we assume that these actions become harder and cost more for larger amounts. Note that this is a reasonable assumption since it is unlikely to reduce the processing time to zero (or making the resource capacity infinite) in a real life system. Thus, we assume that the compression cost function is a convex function of the compression amount and has the same structure as the nervousness cost function.

Recently, Koca et al. (2015) study the stochastic lot sizing problem with controllable processing times and convex compression cost functions. We consider the same compression cost function as that study and apply a similar conic strengthening technique. But, the authors consider the problem under a static uncertainty strategy with $\alpha$ service level constraints, whereas in this paper, we study the multistage stochastic lot sizing problem under a dynamic uncertainty strategy with a different objective function, e.g., we search for a minimum cost production plan that causes less nervousness in the system. Moreover, in this study we exploit the mixing set substructure that arises due to the controllable processing times option.

The effect of new restrictions and controllable processing times on a production schedule is illustrated in the following example.

Example. Consider a 4-period problem instance where the demand of each period is given on the scenario tree in Fig. 1. Assume that all nodes defined for a period have the same probability. For example, probability of realizing the demand represented by one of the nodes in period 3 is $0.25(=1 / 4)$. Suppose that setup cost is 3000 , unit production and inventory holding costs are 5 and 1 for each period, respectively. Cost parameters for each node are obtained by multiplying the corresponding parameter for the period of the node with the node probability. For example, setup, unit production, and inventory holding costs in node 3 are $3000 \times 0.25=750,5 \times 0.25=1.25$, and $1 \times 0.25=0.25$, respectively. Suppose that the capacity of each period is 800 time units and the normal processing time is 1 time unit for each item. Let $x_{i}$ be the production amount in a period if demand represented by node $i$ is realized.

The optimal solution of the classical scenario tree formulation of this problem instance is to produce in the following amounts: $x_{0}=784, x_{2}=212, x_{4}=800, x_{6}=743, x_{7}=88, x_{10}=497, x_{11}=$ $668, x_{13}=800$ and $x_{14}=557$. Total expected cost of this solution is 7875 (for setup) +8010 (for production) +631.5 (for inventory) $=$ 16516.5. Note that according to this solution production decisions depend on the scenario realizations, and there exists a production decision for every period under different scenarios. 


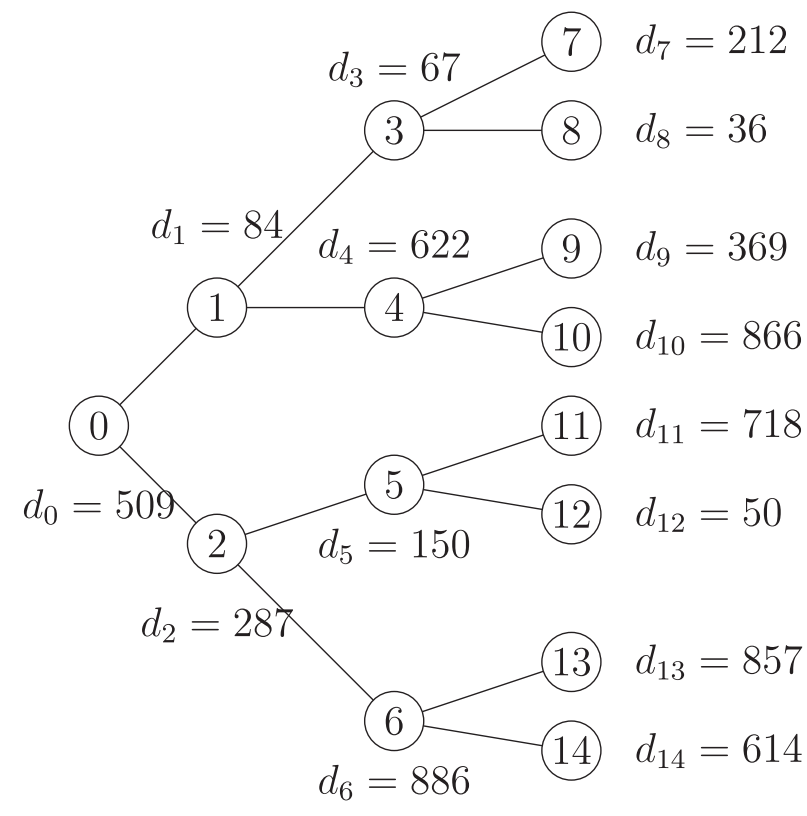

Fig. 1. Example.

Now suppose that setup decisions are taken for each period (not for each node separately) and the production amounts should satisfy the following range constraints :

$$
\begin{array}{cc}
x_{0}=z_{0} & \\
0.2 z_{2} \leq x_{i} \leq 1.8 z_{2} & i=1,2 \\
0.2 z_{3} \leq x_{i} \leq 1.8 z_{3} & i=3, \ldots, 6 \\
0.2 z_{4} \leq x_{i} \leq 1.8 z_{4} & i=7, \ldots, 14
\end{array}
$$

where the new decision variable $z_{t}$ is the promised production amount for period $t$. The solution of this problem is to produce in all periods the following amounts:

$$
x=(509,84,430,89,688,89,800,190,89,303,800,636,89,
$$$$
800,557) \text {. }
$$

The promised production amounts are $z=(509,239,445,445)$. Total expected cost of this solution is 12000 (for setup) +8077.5 (for production) +152.75 (for inventory) $=20230.25$. Note that the total cost is increased due to new restrictions on the production decisions. The cost difference can be conceived as the cost of eliminating setup oriented nervousness and reducing the quantity oriented nervousness.

Now suppose that processing times can be reduced, or capacities can be increased. Assume that processing times can be reduced by at most 0.6 at each period, and the compression cost for each node is given by the function $0.001 \times$ (total compression amount in each node $)^{2}$. In this case the optimal solution is to produce in periods 1 and 3 the following amounts: $x_{0}=$ $796, x_{3}=194, x_{4}=1285, x_{5}=868$, and $x_{6}=1743$; and the optimal promised production amounts are $z_{1}=796$ and $z_{3}=969$. Total expected cost is 6000 (for setup) +9092.5 (for production) +1308.75 (for inventory) +1129.1 (for reducing the processing times $)=17530.35(<20230.25)$. Note that by allowing the controllable processing times, we could produce more in several nodes and eliminate two production periods. In this way, we get rid of the setup cost for two periods, but pay more for inventory and also for reducing the processing times. It can be observed from this example that we add new restrictions to the problem for reducing the system nervousness, and we enlarge the solution space by introducing controllable processing times in this problem context for the first time.
The contributions of this paper are threefold:

- To the best of our knowledge, this is the first study that considers the system nervousness in multistage stochastic lot sizing problem. We propose a new approach to eliminate the setup oriented nervousness and control the quantity oriented nervousness.

- We introduce a new concept called promised production amounts to reduce the quantity oriented nervousness.

- To the best of our knowledge, this is the first study that considers the multistage stochastic lot sizing problem with controllable processing times. We observe the continuous mixing set substructure in our formulation that arises due to this assumption. We reformulate the problem by using the extended formulation for continuous mixing set, and use the valid inequalities developed for mixing sets.

The rest of the paper is organized as follows. In the next section, we review the related literature. In Section 2, we formulate the problem as a SOCMIP and strengthen this formulation by conic strengthening. In Section 3, we show that the continuous mixing set is a relaxation of the lot sizing problem with controllable processing times. We propose extended formulations and valid inequalities based on mixing and continuous mixing set relaxations of our formulation. We test these formulations and inequalities in Section 4, and in Section 5, conclusions and future research directions are discussed.

\section{Literature review}

In this section, we briefly review the related literature.

\subsection{Multistage stochastic lot sizing problem}

When demand follows a finite discrete probability distribution, a scenario tree can be constructed to represent the possible demand realizations for each period. In a scenario tree, each stage corresponds to a time period, and any path from the root node to a leaf node represents a scenario. The dependency between the demand of different periods can be easily formulated in a scenario tree. However, the tree size grows exponentially with the number of possible demand realizations; for example, for 10 periods and 2 possible demand realizations for each period, the number of nodes is 1023. Escudero and Kamesam (1995) consider the multistage stochastic lot sizing problem with two suppliers ((capacitated) inhouse production and (uncapacitated) vendor supply) and different recourse options: simple (production decisions are the same for all scenarios), partial (in-house production decisions are the same for all scenarios) and full (all decisions may be different for different scenarios). The authors assume there is no setup cost and propose a heuristic solution method by clustering the time horizon into three stages: first two stages are periods 1 and 2, respectively, and the remaining time periods are assumed as stage 3 .

Ahmed et al. (2003) consider the multistage stochastic capacity expansion model and draw an equivalence between this problem and the multistage stochastic uncapacitated lot sizing problem. The authors formulate the stochastic uncapacitated lot sizing problem as a facility location problem and show that the WagnerWhitin property (Wagner and Whitin, 1958) does not hold for this problem. Brandimarte (2006) formulates the multi-item stochastic capacitated lot sizing problem as a facility location problem, and develops a fix-and-relax heuristic by partitioning the setup variables according to the time index. Tang et al. (2012) develop a Lagrangian relaxation heuristic for the multistage stochastic lot sizing problem with nonlinear cost functions. Cristobal et al. (2009) consider the stochastic dynamic programming approach to solve large 
scale planning problems, and the authors test their approach on the multistage stochastic lot sizing problem instances.

Guan et al. (2006b) develop a family of valid inequalities, called $\left(Q, S_{Q}\right)$, for the stochastic uncapacitated lot sizing problem and Guan et al. (2006a) show that these inequalities are sufficient for describing the convex hull of the set of solutions for the two period problem. Di Summa and Wolsey (2008) prove that these inequalities are also mixing inequalities and extend these results to the constant capacitated case. Guan et al. (2009) develop valid inequalities employed in a branch-and-cut method for a multistage (capacitated) stochastic lot sizing problem.

Halman et al. (2009) show that multistage stochastic lot sizing problem is NP-hard when the problem is uncapacitated, production and inventory costs are linear and there are two possible demand scenarios for each period. Guan (2011) develop polynomial time (in the tree size) dynamic programming algorithms for the problem when backlogging is possible and/or capacities are varying between periods.

\subsection{System nervousness}

System nervousness is caused by the changes in the production plans. Nervousness at an upper level of the supply chain affects all the supply chain and it causes lack of coordination in the production systems. If the system is not flexible, i.e., if revisions cannot be handled easily, nervousness becomes a bigger problem and in these systems, it may be more appropriate to look for a more stable production plan, in which revisions are not needed (Heisig, 2001).

There are few studies in the literature that consider nervousness. In early studies on this subject, simulation of the systems is used to test different strategies and to investigate the impact of parameter settings (Blackburn et al., 1986; Kadipasaoglu and Sridharan, 1997). Kropp et al. (1983) incorporate nervousness to the total cost function and solve the problems heuristically. Inderfurth (1994), De Kok and Inderfurth (1997), and Heisig (2001) consider system nervousness caused by inventory policies such as $(s, S),(s, n Q),(R, S)$ policies and develop different measures for nervousness. However, in all of these studies, the systems are assumed as stationary. According to these studies, the $(s, S)$ policy, which is the optimal policy for stationary systems (Scarf, 1960), performs worst in terms of system nervousness, since a production decision for each period is taken at the beginning of that period according to the revised inventory level. Pujawan (2004) considers a case study of nervousness in a manufacturing company and emphasizes that nervousness is an important issue in practice.

In recent studies, new nervousness measures are considered for nonstationary systems. Kilic and Tarim (2011) develop a method for measuring cost of system nervousness in nonstationary systems under $(S, S)$ and $(R, S)$ policies and conclude that the $(R, S)$ policy performs better in terms of nervousness. Tunc et al. (2016) develop an MIP formulation for the lot sizing problem under a generalized $(R, S)$ policy where the ordering cost functions are piecewise concave. Tunc et al. (2013) introduce a method for evaluating the costs of setup and quantity oriented nervousness by comparing static, dynamic and static-dynamic uncertainty strategies. Note that static uncertainty strategy is nervousness free since all the production decisions are taken at the beginning of the planning horizon. On the other hand, the dynamic uncertainty strategy causes both setup oriented and quantity oriented nervousness. As a combination of these two strategies, static-dynamic uncertainty strategy causes only quantity oriented nervousness. The authors conclude that, setup oriented nervousness can be avoided by a small cost increase in the system whereas it is harder to avoid quantity oriented nervousness.
A rolling horizon is frequently applied to the systems when it is not possible to have an accurate forecast for the demand of further periods (Baker, 1977). Determining the length of the planning interval is one of the issues of the rolling horizon approach. As a production plan for a given period may change as much as this interval length, this method may cause system nervousness (Inderfurth, 1994). Simpson (2001) compares several lot sizing rules with respect to different aspects including nervousness in extensive rolling horizon simulation tests. Kazan et al. (2000) evaluate different algorithms under the rolling horizon when there exist cost terms associated with setup and quantity oriented nervousness.

In this study, we restrict quantity oriented nervousness by imposing range constraints on the production decisions. The idea is very similar to the restricted recourse concept of Vladimirou and Zenios (1997). Vladimirou and Zenios (1997) search for recourse robust solutions for two stage stochastic linear programs by investigating different formulations in which variability of the second stage decisions is restricted via some additional constraints. The authors develop solution procedures for these formulations by using the primal-dual interior point method.

\subsection{Controllable processing times}

Controllable processing times are well studied in the context of scheduling. Earlier studies on this subject assume linear compression costs, although as it is stated in recent studies, in many applications reducing the processing times gets harder (and more expensive) as the compression amount increases (Aktürk et al., 2009). Thus, considering a convex compression cost function is more realistic since a convex function represents increasing marginal costs and may limit higher usage of the resource due to environmental issues. A detailed review on scheduling with controllable processing times can be found in Shabtay and Steiner (2007).

As reducing the processing time of a job is equivalent to increasing the production capacity, controllable processing times can be seen as an alternative to subcontracting or overloading. There are studies in the literature that consider the lot sizing problem with subcontracting or outsourcing (Atamtürk and Hochbaum, 2001; Helber et al., 2013; Merzifonluoğlu et al., 2007). However, in all of these studies costs of these options are assumed as either linear or concave. Koca et al. (2015) is the only study that considers the lot sizing problem with convex compression cost functions.

\section{Problem definition and formulation}

Suppose that demand follows a discrete distribution and possible demand realizations are represented by a scenario tree $\mathcal{T}=$ $(V, E)$ with $T$ stages and $n$ nodes. Let $t(i)$ be the time period of node $i \in V$ and $\pi_{i}$ be the probability associated with the state represented by node $i$. The set of nodes defined for period $t$ is denoted by $V_{t}$. The unique predecessor of node $i$ is given by $i^{-}$and demand represented by node $i$ is denoted by $d_{i}$. Assume that 0 is the root node. Let $P(i, j)$ be the path from node $i$ to node $j$ and $V(u)$ be the set of descendants of node $u$, including $u$.

Let $f_{t}$ be the setup cost for period $t$, and $c_{i}$ and $h_{i}$ be the unit production and inventory holding costs for node $i$, respectively. We assume that all the cost parameters defined for each node include the probability of the node. For example, if the probability of realizing the demand represented by node $i$ is 0.5 and the unit production cost for period $t(i)$ is 2 units, then we assume that the unit production cost for that node is $c_{i}=2 \times 0.5=1$. But note that as the setup decisions are taken for periods, not for nodes separately, setup costs do not include node probabilities. 


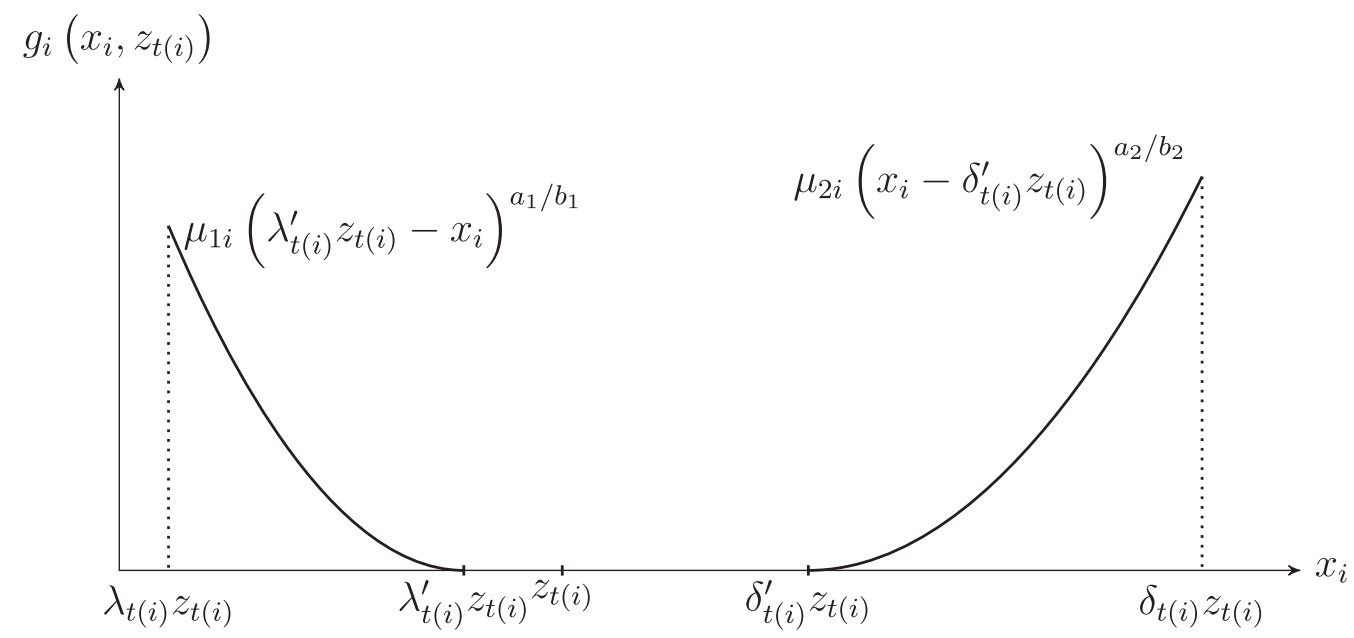

Fig. 2. Cost of nervousness.

We assume that the capacity in period $t$ is $C_{t}$ time units. Processing time without any compression is $p$ time units, and processing time of any item can be reduced by at most $\beta(<p)$ time units. Without loss of generality, we assume that $p=1$. If processing times in node $i$ are decreased by $r_{i}$ time units in total, then the compression cost is given by $\gamma_{i}\left(r_{i}\right)=\kappa_{i} r_{i}^{e / d}$ where $\kappa_{i} \geq 0$ and $e \geq d>0$. Note that $\gamma$ is a convex function of the compression amount $r$ and this function can represent the increasing marginal cost of decreasing the processing times in larger amounts.

In the classical scenario tree formulation of the lot sizing problem, even though optimal production decisions for each node are known, we may not know the exact production amount in a period until the demand of the period is realized. This situation causes both setup and quantity oriented nervousness in the system. In this study, we want to find a minimum cost solution for the problem which results in less nervousness in the system. In other words, we have two different objectives: we still want to find a minimum cost production plan for the system and we want to decrease the system nervousness by considering additional constraints on the production decisions.

In order to reduce the setup oriented nervousness, we consider setup decisions for periods rather than deciding for each node separately. Thus, we determine the production periods at the beginning of the planning horizon, and in this way we eliminate the setup oriented nervousness from our formulation. Moreover, we control quantity oriented nervousness by restricting production amounts under different scenarios and penalizing different production decisions for the same time period. We define two types of decisions related to production amounts (exact production amounts for each node and promised production amounts for each period), and assume that there is a nervousness cost depending on the relation of these two decisions.

Let $y_{t}$ be the setup variable which is equal to 1 if there exists a production in period $t$ and 0 otherwise. Let $x_{i}$ be the production amount in node $i$ and $z_{t}$ be the promised production amount in period $t$. We relate the promised production amount for a period and the production decisions for each node defined for that period by the range parameters $\lambda_{t} \leq 1$ and $\delta_{t} \geq 1: \lambda_{t} z_{t} \leq x_{i} \leq \delta_{t} z_{t}$ for $i \in V_{t}$. In other words, the production amount of any node defined for period $t$ should be in the interval $\left[\lambda_{t} z_{t}, \delta_{t} z_{t}\right]$. The inequalities can be rewritten as $\lambda_{t(i)} z_{t(i)} \leq x_{i} \leq \delta_{t(i)} z_{t(i)}$ for $i \in V$.

Accuracy of promised production amounts $(z)$ is controlled by the parameters $\lambda_{t}$ and $\delta_{t}$. When these parameters get close to 1 , the interval $\left[\lambda_{t} z_{t}, \delta_{t} z_{t}\right]$ shrinks and $z_{t(i)}$ becomes closer to the exact production/order amount $x_{i}$. Moreover, in this case, production amounts for different nodes also get closer to each other and quantity oriented nervousness decreases since $\lambda_{t} z_{t} \leq x_{i} \leq \delta_{t} z_{t}$ should hold for all $i \in V_{t}$.

Suppose that $g_{i}\left(x_{i}, z_{t(i)}\right)$ denotes the nervousness (or penalty) cost for node $i$ given that $x_{i}$ units are produced in node $i$ and $z_{t(i)}$ units are promised to be produced in period $t(i)$. To be more general, we assume the system is flexible enough for letting the production amount in the interval $\left[\lambda_{t}^{\prime} z_{t}, \delta_{t}^{\prime} z_{t}\right]$ without any penalty cost where $\lambda_{t} \leq \lambda_{t}^{\prime} \leq 1$ and $1 \leq \delta_{t}^{\prime} \leq \delta_{t}$. In other words, we assume that if $x_{i} \in\left[\lambda_{t}^{\prime} z_{t}, \delta_{t}^{\prime} z_{t}\right]$ then no nervousness cost is incurred. But if the production amount is not in this range, then a nervousness cost which is a convex function of the minimum distance between the production amount and this interval is incurred. Note that, we can prevent larger distances between each production amount and this interval by a convex nervousness cost function. To this end, we define $x_{1 i}$ and $x_{2 i}$ as $x_{1 i}=\left[\lambda_{t(i)}^{\prime} z_{t(i)}-x_{i}\right]^{+}$and $x_{2 i}=\left[x_{i}-\delta_{t(i)}^{\prime} z_{t(i)}\right]^{+}$ where $[a]^{+}=\max \{a, 0\}$ and assume that the nervousness cost is given by the function

$g_{i}\left(x_{i}, z_{t(i)}\right)=\mu_{1 i} x_{1 i}^{a_{1} / b_{1}}+\mu_{2 i} x_{2 i}^{a_{2} / b_{2}}$

where $\mu_{1 i}, \quad \mu_{2 i} \geq 0$ are nervousness cost coefficients, and $a_{1} \geq b_{1}>0, \quad a_{2} \geq b_{2}>0$. The cost function $g_{i}$ is illustrated in Fig. 2 .

We assume that $\lambda^{\prime}, \delta^{\prime}$, and the cost parameters are determined according to the flexibility of the system. For example, if the system is very sensitive to changes in the production amounts, then it is appropriate to set $\lambda^{\prime}$ and $\delta^{\prime}$ close to 1 , and $\mu_{1 i}, \mu_{2 i}, a_{1} / b_{1}, a_{2} / b_{2}$ to large values. On the other hand, if it is easy to adapt the system for changes, then one can set $\lambda^{\prime}=\lambda, \delta^{\prime}=\delta$ and get rid of the nervousness cost. Thus, we cover various special cases for different parameter settings, and we will explore some of these special cases in Section 5.

In our formulation we control and reduce system nervousness in different ways. Since we take setup decisions for each period, independent from scenario realizations, we exclude setup oriented nervousness. Note that, if $\lambda_{t(i)}=0$, it is still possible to have a solution where $y_{t(i)}=1$ but $x_{i}=0$, but the converse is not possible. Furthermore, due to the promised production amounts, a set of newly introduced constraints, and parameters $\lambda$ and $\delta$, we keep the production decisions under different scenarios within some certain range of the promised production amounts. Still we might obtain different production decisions under different scenarios, but we guarantee that the amounts are within some predetermined range of the promised production amounts whose values are known beforehand. Finally, by penalizing the differences 
between the promised production amounts and the actual production decisions, we reduce quantity oriented nervousness.

In addition to the variables defined above, let $s_{i}$ be the inventory on hand at the end of period $t(i)$ for node $i$. We assume that all the realized demand should be satisfied, thus backordering or shortages are not allowed. Note that, we have three types of recourse actions to hedge against demand uncertainty: production, inventory, and compression amounts are defined for each node separately.

The problem can be formulated as the following:

$$
\begin{aligned}
\min & \sum_{t=1}^{T} f_{t} y_{t}+\sum_{i \in V}\left(c_{i} x_{i}+h_{i} s_{i}\right)+\sum_{i \in V}\left(\mu_{1 i} x_{1 i}^{a_{1} / b_{1}}+\mu_{2 i} x_{2 i}^{a_{2} / b_{2}}\right) \\
& +\sum_{i \in V} \kappa_{i} r_{i}^{e / d}
\end{aligned}
$$

$$
\begin{aligned}
& \text { s.t. } s_{i^{-}}+x_{i}=d_{i}+s_{i} \quad i \in V \\
& x_{i} \leq C_{t(i)} y_{t(i)}+r_{i} \quad i \in V \\
& r_{i} \leq \beta x_{i} \quad i \in V \\
& \lambda_{t(i)} z_{t(i)} \leq x_{i} \leq \delta_{t(i)} z_{t(i)} \quad i \in V \\
& x_{1 i} \geq \lambda_{t(i)}^{\prime} z_{t(i)}-x_{i} \quad i \in V \\
& x_{2 i} \geq x_{i}-\delta_{t(i)}^{\prime} z_{t(i)} \quad i \in V \\
& s_{0^{-}}=0 \\
& x_{i}, x_{1 i}, x_{2 i}, s_{i}, \quad r_{i} \geq 0 \quad i \in V \\
& y_{t} \in\{0,1\}, \quad z_{t} \geq 0 \quad t=1, \ldots, T
\end{aligned}
$$

In the objective function (1), we minimize the total (expected) setup, production, inventory holding, nervousness and compression costs. Constraints (2) are the classical demand satisfaction (inventory balance) constraints for each node $i$ : total amount due to production in node $i$, and inventory left from the unique parent of node $i$ should be equal to the total demand in node $i$, and final inventory level at node $i$. We assume that the initial inventory $s_{0^{-}}$ is zero by constraints (8). Due to constraints (3), total time to produce $x_{i}$ units $\left(x_{i}\right.$ time units since we assume $\left.p=1\right)$ minus the total compression amount in node $i$ ( $r_{i}$ time units) should be less than or equal to the capacity. Constraints (4) ensure that the processing time of each job is reduced by at most $\beta$ time units. Constraints (5) are the range constraints that relate the variables $x_{i}$ and $z_{t(i)}$, and also the production amounts of different nodes defined for the same time period $t(i)$. Note that, according to these constraints, if nodes $i$ and $i^{\prime}$ are both defined for period $t$, then production amounts for both of these nodes should be in the same range: $x_{i}, x_{i^{\prime}} \in\left[\lambda_{t} z_{t}, \delta_{t} z_{t}\right]$ for $i, i^{\prime} \in V_{t}$. Definitions of the variables $x_{1 i}$ and $x_{2 i}$ are expressed in the constraints (6) and (7) (and nonnegativity constraints), respectively. Constraints (9) and (10) define the ranges and types of the variables.

Note that, the formulation LSI has a nonlinear objective function due to the compression and nervousness costs. In the next section, we will reformulate the problem as a SOCMIP and strengthen the formulation so that it can be solved by a commercial solver.

\section{Reformulation of the problem}

We now reformulate LSI as a SOCMIP. To do this, we first introduce nonnegative auxiliary variables $w_{1 i}, w_{2 i}$ and $v_{i}$ such that

$x_{1 i}^{a_{1} / b_{1}} \leq w_{1 i} \quad i \in V$

$x_{2 i}^{a_{2} / b_{2}} \leq w_{2 i} \quad i \in V$

$r_{i}^{e / d} \quad \leq v_{i} \quad i \in V$

We can replace nonlinear terms in the objective function (1) by these auxiliary variables, and add inequalities (11)-(13) to formulation LSI. Moreover, as $b_{1}, b_{2}, d>0$, and $y_{t(i)}=0$ implies $x_{i}=x_{1 i}=$ $x_{2 i}=r_{i}=0$, we can multiply the right hand sides of inequalities (11)-(13) by $y_{t}$ :

$x_{1 i}^{a_{1}} \leq w_{1 i}^{b_{1}} y_{t(i)}^{a_{1}-b_{1}} \quad i \in V$

$x_{2 i}^{a_{2}} \leq w_{2 i}^{b_{2}} y_{t(i)}^{a_{2}-b_{2}} \quad i \in V$

$r_{i}^{e} \leq v_{i}^{d} y_{t(i)}^{e-d} \quad i \in V$

Note that, if there is no production in period $t$, then $y_{t}=0$ and $x_{i}, x_{1 i}, x_{2 i}, r_{i}$ will be equal to zero; and if $y_{t}=1$, then inequalities (14)-(16) are equivalent to (11)-(13). This procedure is called "conic strengthening" by Aktürk et al. (2009) since it strengthens the continuous relaxation, and the resulting inequalities can be represented by conic quadratic inequalities.

As given in Ben-Tal and Nemirovski (2001), for $l \in \mathbb{Z}_{+}$and $\zeta, \xi_{1}, \ldots, \xi_{2^{l}} \geq 0$, the inequality $\zeta^{2^{l}} \leq \xi_{1} \ldots \xi_{2^{l}}$ can be represented by using $O\left(2^{l}\right)$ variables and hyperbolic inequalities of the form $v \leq \omega_{1} \omega_{2}$ which is conic quadratic representable:

$\left\|\left(\begin{array}{c}2 v \\ \omega_{1}-\omega_{2}\end{array}\right)\right\| \leq \omega_{1}+\omega_{2}$.

Using these results, one can show that inequalities (14)-(16) can be represented by $O\left(\log _{2}\left(a_{1}\right)\right), O\left(\log _{2}\left(a_{2}\right)\right), O\left(\log _{2}(e)\right)$ variables and conic quadratic constraints, respectively (Aktürk et al., 2009).

Thus, LSI can be reformulated as

$$
\begin{aligned}
\min & \sum_{t=1}^{T} f_{t} y_{t}+\sum_{i \in V}\left(c_{i} x_{i}+h_{i} s_{i}\right)+\sum_{i \in V}\left(\mu_{1 i} w_{1 i}+\mu_{2 i} w_{2 i}\right) \\
& +\sum_{i \in V} \kappa_{i} v_{i} \\
\text { s.t. } & (2)-(10) \\
& (14)-(16) \\
& w_{1 i}, w_{2 i}, v_{i} \geq 0 \quad i \in V
\end{aligned}
$$

LSII has a linear objective function, and nonlinear constraints (14)-(16), which are conic quadratic representable. We will refer to the conic quadratic representation of LSII as CLSII. In CLSII, we replace inequalities (14)-(16) with their conic quadratic representations, and obtain a quadratically constrained MIP with linear objective function, which can be solved by fast algorithms of commercial solvers like IBM ILOG CPLEX.

\section{Valid inequalities}

Note that our problem is a generalization of the stochastic capacitated lot sizing problem. The capacity constraints (3) are relaxations of the classical capacity constraints due to the controllable processing times option. Therefore, valid inequalities developed for 
the stochastic capacitated lot sizing problem may not be valid for our problem, except the ones valid for the stochastic uncapacitated lot sizing problem (e.g. Guan et al., 2006b). But, it is possible to derive new valid inequalities for our problem by using the new variables and constraints introduced. In this section, we derive new valid inequalities for the formulation LSII.

For $u \in V$ and $k \in V(u)$, if balance constraints (2) are summed for $i \in P(u, k)$, we obtain the following inequalities:

$s_{u^{-}}+\sum_{i \in P(u, k)} x_{i}=d_{u k}+s_{k} \quad k \in V(u)$

where $d_{u k}=\sum_{i \in P(u, k)} d_{i}$. Since $s \geq 0$,

$s_{u^{-}}+\sum_{i \in P(u, k)} x_{i} \geq d_{u k} \quad k \in V(u)$.

For the classical capacitated lot sizing problem, capacity is an upper bound for the production amount: $x_{i} \leq C_{t(i)}$. But when the processing times are controllable, we can produce more than the capacity by reducing the processing times. So for our problem, an upper bound for the production amount is $C_{t(i)} y_{t(i)}+r_{i}$. Due to these upper bounds, inequalities (20) imply

$s_{u^{-}}+\sum_{i \in P(u, k)} r_{i}+\sum_{i \in P(u, k)} C_{t(i)} y_{t(i)} \geq d_{u k} \quad k \in V(u)$.

In this section, we assume that capacities are the same for all periods, $C_{t}=\bar{C}$ for $t=1, \ldots, T$ and demand is normalized with respect to the capacity, i.e., $\bar{d}_{i}=\frac{d_{i}}{\bar{C}}$. The validity of the inequalities derived in this section depends on this assumption. If the capacities are time-variant, one can take $\bar{C}=\max _{t}\left\{C_{t}\right\}$, and use the valid inequalities. But, obviously, the quality of the inequalities depends on the tightness of $\bar{C}$, i.e., if the difference between $\bar{C}$ and $\min _{t} C_{t}$ is very large, then the inequalities may not perform well.

So, inequalities (21) are rewritten as

$s_{u^{-}}+\sum_{i \in P(u, k)} r_{i}+\sum_{i \in P(u, k)} y_{t(i)} \geq \bar{d}_{u k} \quad k \in V(u)$.

Note that, in these inequalities the variables are also expressed in terms of capacity (for example $\bar{r}_{i}=\frac{r_{i}}{\bar{C}}$ ), but for ease of notation we do not rename them.

We will derive two different sets of valid inequalities for the problem. First, we will show that for given $u$, inequalities (22) define a continuous mixing set. Van Vyve (2005) introduced valid inequalities and an extended formulation for the continuous mixing set and showed that these inequalities are sufficient for describing the convex hull of the set. We will make use of this study to derive valid inequalities for our problem. Next, we will apply the mixing scheme of Günlük and Pochet (2001) to inequalities (22) to obtain valid inequalities for our formulation.

\subsection{Continuous mixing set structure}

Now we will show that for given $u$, inequalities (22) define a continuous mixing set. Let $\alpha_{k}=\sum_{i \in P(u, k)} y_{t(i)}-\left\lfloor\bar{d}_{u k}\right\rfloor, \sigma_{k}=$ $\sum_{i \in P(u, k)} r_{i}, \hat{d}_{k}=\bar{d}_{u k}-\left\lfloor\bar{d}_{u k}\right\rfloor$ for $k \in V(u)$, and $s=s_{u^{-}}$. Then, inequalities (22) are equivalent to

$$
\begin{array}{cl}
s+\sigma_{k}+\alpha_{k} \geq \hat{d}_{k} & k \in V(u) \\
\sigma_{k} \in \mathbb{R}_{+}, \alpha_{k} \in \mathbb{Z} & k \in V(u) \\
s \in \mathbb{R}_{+} &
\end{array}
$$

Valid inequalities of Van Vyve (2005) for the continuous mixing set are based on costs of cycles in a graph constructed as follows. Let $V^{\prime}=\left\{j_{0}, j_{1}, \ldots, j_{|V(u)|}\right\}$ be an ordered set including all the descendants of node $u$ such that $\hat{d}_{j_{i}} \leq \hat{d}_{j_{i+1}}$ for $i=$ $1, \ldots,|V(u)|-1$. In this set, $j_{0}$ is a dummy node with $\hat{d}_{j_{0}}=$
0 . Let $G_{u}^{\prime}=\left(V^{\prime}, A^{\prime}\right)$ be a graph defined on the node set $V^{\prime}$ with the arc set $A^{\prime}=\left\{\left(j_{0}, j_{i}\right): 1 \leq i \leq|V(u)|\right\} \cup\left\{\left(j_{i}, j_{0}\right): 1 \leq i \leq\right.$ $|V(u)|\} \cup\left\{\left(j_{i}, j_{l}\right): \hat{d}_{j_{i}} \neq \hat{d}_{j_{l}}, 1 \leq i, l \leq|V(u)|\right\}$. Suppose that cost of an $\operatorname{arc}\left(j_{i}, j_{l}\right) \in A^{\prime}$ is given by:

$$
\Phi_{j_{i}, j_{l}}(s, \sigma, \alpha)= \begin{cases}s+\sigma_{j_{i}}+\left(\hat{d}_{j_{i}}-\hat{d}_{j_{l}}+1\right) \alpha_{j_{i}}-\hat{d}_{j_{l}} & \text { if } 1 \leq i<l \leq|V(u)|, \\ \sigma_{j_{i}}+\left(\hat{d}_{j_{i}}-\hat{d}_{j_{l}}\right) \alpha_{j_{i}} & \text { if } 1 \leq l<i \leq|V(u)|, \\ s+\sigma_{j_{i}}+\alpha_{j_{i}}-\hat{d}_{j_{i}} & \text { if } i=l \text { and } 1 \leq i \leq|V(u)|, \\ s-\hat{d}_{j_{l}} & \text { if } i=0 \text { and } 1 \leq l \leq|V(u)|, \\ \sigma_{j_{i}}+\hat{d}_{j_{i}} \alpha_{j_{i}} & \text { if } l=0 \text { and } 1 \leq i \leq|V(u)| .\end{cases}
$$

Graph $G_{u}^{\prime}$ should not include a negative cycle. Thus, each cycle in this graph leads to a valid inequality for the set defined by inequalities (22). For a given elementary cycle $\mathcal{C} \subset A^{\prime}$, the associated cycle inequality is

$\sum_{\left(j_{i}, j_{l}\right) \in \mathcal{C}} \Phi_{j_{i}, j_{l}}(s, \sigma, \alpha) \geq 0$.

Separation of these valid inequalities is equivalent to finding a negative cost cycle in the graph $G_{u}^{\prime}$ and this can be done in $O\left(|V(u)|^{3}\right)$ time by the shortest path algorithm of Floyd-Warshall (Ahuja et al., 1993). Moreover, Van Vyve (2005) developed an extended formulation for the continuous mixing set based on the dual of this separation problem. Note that the graph $G_{u}^{\prime}$ does not contain any negative cycle if there exists $\theta \in \mathbb{R}^{|V(u)|+1}$ such that

$\Phi_{j_{i}, j_{l}}(s, \sigma, \alpha) \geq \theta_{j_{i}}-\theta_{j_{l}}$ for all $\left(j_{i}, j_{l}\right) \in A^{\prime}$

Inequalities (24) are equivalent to:

$$
\begin{array}{r}
s+\sigma_{j_{i}}+\left(\hat{d}_{j_{i}}-\hat{d}_{j_{l}}+1\right) \alpha_{j_{i}} \geq \hat{d}_{j_{l}}+\theta_{j_{i}}-\theta_{j_{l}} \\
\left(j_{i}, j_{l}\right) \in A^{\prime}: 1 \leq i<l \leq|V(u)|
\end{array}
$$

$\sigma_{j_{i}}+\left(\hat{d}_{j_{i}}-\hat{d}_{j_{l}}\right) \alpha_{j_{i}} \geq \theta_{j_{i}}-\theta_{j_{l}} \quad\left(j_{i}, j_{l}\right) \in A^{\prime}: 1 \leq l<i \leq|V(u)|$

$s+\sigma_{j_{i}}+\alpha_{j_{i}} \geq \hat{d}_{j_{i}} \quad 1 \leq i \leq|V(u)|$

$s \geq \hat{d}_{j_{l}}+\theta_{j_{0}}-\theta_{j_{l}}$

$$
1 \leq l \leq|V(u)|
$$

$\sigma_{j_{i}}+\hat{d}_{j_{i}} \alpha_{j_{i}} \geq \theta_{j_{i}}-\theta_{j_{0}}$

$$
1 \leq i \leq|V(u)|
$$

Note that the extended formulation given by inequalities (25)(29) and the valid inequalities (23) are derived for a fixed node $u$ and for all the descendants of node $u$. In our computational experiments, we consider the extended formulation for each $u \in V$ and added the extended formulation to our formulation. Results for this experiment are given in Section 6 .

\subsection{Mixing set structure}

In this subsection, we will apply the mixing procedure of Günlük and Pochet (2001) to inequalities (22):

$s_{u^{-}}+\sum_{i \in P(u, k)} r_{i}+\sum_{i \in P(u, k)} y_{t(i)} \geq \bar{d}_{u k} \quad k \in V(u)$.

Suppose that $u$ is fixed and let $\hat{d}_{k}=\bar{d}_{u k}-\left\lfloor\bar{d}_{u k}\right\rfloor$ be the fractional part of the total demand from node $u$ to node $k$. Let $R=$ $\left\{i_{1}, \ldots, i_{K}\right\} \subseteq V(u)$ be an ordered set such that $0=\hat{d}_{i_{0}} \leq \hat{d}_{i_{1}} \leq \hat{d}_{i_{2}} \leq$ $\ldots \leq \hat{d}_{i_{K}}$. Set of nodes in the paths from node $u$ to the nodes in $R$ are given by $V_{R}=\cup_{k \in R} P(u, k)$. Note that

$s=s_{u^{-}}+\sum_{i \in V_{R}} r_{i} \geq s_{u^{-}}+\sum_{i \in P(u, k)} r_{i} \quad \forall k \in R$. 
Thus, $s$ is an upper bound for the continuous part of inequalities (22) for the nodes in $R$ and inequalities (22) imply

$s+\sum_{i \in P(u, k)} y_{t(i)} \geq \bar{d}_{u k} \quad k \in R$.

If we define $y_{u k}=\sum_{i \in P(u, k)} y_{t(i)}$ for $k \in R$, then inequalities (30) can be rewritten as

$s+y_{u k} \geq \bar{d}_{u k} \quad k \in R$.

Inequalities (31) define a mixing set since $s \in \mathbb{R}$ and $y_{u k} \in \mathbb{Z}$ for $k \in R$. We will apply the mixing procedure to these inequalities to obtain valid inequalities for our problem. Mixing of inequalities (31) leads to the following inequalities

$s \geq \sum_{j=1}^{K}\left(\hat{d}_{i_{j}}-\hat{d}_{i_{j-1}}\right)\left(\left\lfloor\bar{d}_{u, i_{j}}\right\rfloor+1-y_{u, i_{j}}\right)$

$s \geq \sum_{j=1}^{K}\left(\hat{d}_{i_{j}}-\hat{d}_{i_{j-1}}\right)\left(\left\lfloor\bar{d}_{u, i_{j}}\right\rfloor+1-y_{u, i_{j}}\right)+\left(1-\hat{d}_{i_{K}}\right)\left(\left\lfloor\bar{d}_{u, i_{1}}\right\rfloor-\bar{y}_{u, i_{1}}\right)$

Let $\bar{t}_{R}$ be the maximum time period for the nodes in the set $R$, i.e., $\bar{t}_{R}=\max \{t(i): i \in R\}$. For $t=t(u), \ldots, \bar{t}_{R}$, define

$\Delta_{t}(R)=\sum_{i_{j} \in R: t\left(i_{j}\right) \geq t}\left(\hat{d}_{i_{j}}-\hat{d}_{i_{j-1}}\right)$.

Consequently, inequalities (32) and (33) are equivalent to

$$
\begin{aligned}
& s_{u^{-}}+\sum_{i \in V_{R}} r_{i}+\sum_{t=t(u)}^{\bar{t}_{R}} \Delta_{t}(R) y_{t} \geq \sum_{j=1}^{K}\left(\hat{d}_{i_{j}}-\hat{d}_{i_{j-1}}\right)\left(\left\lfloor\bar{d}_{u, i_{j}}\right\rfloor+1\right) \\
& s_{u^{-}}+\sum_{i \in V_{R}} r_{i}+\sum_{t=t(u)}^{t\left(i_{1}\right)}\left(\Delta_{t}(R)+1-\hat{d}_{i_{K}}\right) y_{t}+\sum_{t=t\left(i_{1}\right)+1}^{\bar{t}_{R}} \Delta_{t}(R) y_{t} \\
& \geq \sum_{j=1}^{K}\left(\hat{d}_{i_{j}}-\hat{d}_{i_{j-1}}\right)\left(\left\lfloor\bar{d}_{u, i_{j}}\right\rfloor+1\right)+\left(1-\hat{d}_{i_{K}}\right)\left\lfloor\bar{d}_{u, i_{1}}\right\rfloor
\end{aligned}
$$

Inequalities (34) and (35) are valid for our problem and can be separated in $O(n \log n)$ time by using the separation scheme described in Pochet and Wolsey (2006). Note that these inequalities are derived for fixed $u$, and in our computational experiments given in Section 6, we consider these inequalities for all $u \in V$.

\section{Computational experiments}

In this section, we first test the valid inequalities developed in the previous section, and then we investigate the nervousness caused by the production schedules obtained by our formulation.

Note that, there are several parameters for controlling the nervousness. Different values of $\lambda^{\prime}, \lambda, \delta, \delta^{\prime}$ and different forms of the nervousness cost function $g$ may lead to different problem settings:

S1. If $\lambda^{\prime}=\lambda$ and $\delta^{\prime}=\delta$, then the nervousness cost is equal to 0 . Thus, we obtain a formulation with no nervousness cost function. But note that, with $\lambda$ and $\delta$ parameters, it is still possible to decrease quantity oriented nervousness by controlling range of the production amounts by constraints (5).

S2. If $\lambda_{t}=0$ and $\delta_{t}$ is sufficiently large, i.e., $\delta_{t}=\frac{C_{t}}{p-u}$, for all $t$, then the range constraints (5) become redundant. Thus, we obtain a formulation without range constraints. But, we can still penalize the differences between $x_{i}$ and $z_{t}$ by the nervousness cost function.
S3. If $\lambda_{t}=\lambda_{t}^{\prime}=0, \delta_{t}=\delta_{t}^{\prime}$ and $\delta_{t}$ is sufficiently large, i.e., $\delta_{t}=$ $\frac{C_{t}}{p-u}$, then the formulation is equivalent to the static - dynamic uncertainty strategy as production periods are determined beforehand and the production amount of each period is determined as an answer to the demand realization. In this case, variables $z_{t(i)}, x_{1 i}, x_{2 i}$ and constraints (5) - (7) can be ignored.

S4. If $\lambda_{t}=\lambda_{t}^{\prime}=\delta_{t}=\delta_{t}^{\prime}=1$ for all $t$, then the formulation is equivalent to static uncertainty strategy since production decision for a period is the same regardless of the demand realization $\left(x_{i}=z_{t(i)}\right.$ for all $\left.i \in V_{t}\right)$.

55. If $a_{1}=b_{1}$ and $a_{2}=b_{2}$, then the nervousness cost is a piecewise linear function (with at most three pieces): $g_{i}\left(x_{i}, z_{t(i)}\right)=\mu_{1 i}\left[\lambda_{t(i)}^{\prime} z_{t(i)}-x_{i}\right]^{+}+\mu_{2 i}\left[x_{i}-\delta_{t(i)}^{\prime} z_{t(i)}\right]^{+} \quad$ for $x_{i} \in\left[\lambda_{t(i)} z_{t(i)}, \delta_{t(i)} z_{t(i)}\right]$ (see Fig. 3(i)).

S6. If $a_{1}=b_{1}, a_{2}=b_{2}$ and $\lambda_{t(i)}^{\prime}=\delta_{t(i)}^{\prime}=1$, then the nervousness cost is a piecewise linear function (with at most two pieces): $g_{i}\left(x_{i}, z_{t(i)}\right)=\mu_{1 i}\left[z_{t(i)}-x_{i}\right]^{+}+\mu_{2 i}\left[x_{i}-z_{t(i)}\right]^{+}$for $x_{i} \in\left[\lambda_{t(i)} z_{t(i)}\right.$, $\left.\delta_{t(i)} z_{t(i)}\right]$ (see Fig. 3(ii)).

S7. If $\lambda_{t(i)}^{\prime}=\delta_{t(i)}^{\prime}=1$, then the nervousness cost function is given by $g_{i}\left(x_{i}, z_{t(i)}\right)=\mu_{1 i}\left(\left[z_{t(i)}-x_{i}\right]^{+}\right)^{a_{1} / b_{1}}+\mu_{2 i}\left(\left[x_{i}-\right.\right.$ $\left.\left.z_{t(i)}\right]^{+}\right)^{a_{2} / b_{2}}$ for $x_{i} \in\left[\lambda_{t(i)} z_{t(i)}, \delta_{t(i)} z_{t(i)}\right]$ (see Fig. 3(iii)).

S8. The general setting of the nervousness cost function with $\lambda_{t} \leq \lambda_{t}^{\prime} \leq 1$ and $1 \leq \delta_{t}^{\prime} \leq \delta_{t}$ (see Fig. 2).

In our computational experiments, we refer to these settings. We assume that the compression cost function is quadratic, and $m=2$ (number of children of each node), $\beta=0.6, d_{i} \sim U[1,1000]$, $c_{i} \sim \pi_{i} U[20,30], h_{i} \sim \pi_{i} U[1,10], \kappa_{i} \sim \pi_{i} U[0.04 \bar{\kappa}, 0.06 \bar{\kappa}]$ for $i \in V$; $f_{t} \sim U[4 \bar{f}, 6 \bar{f}]$, and $C_{t}=\bar{c}(T-1)$ for $t=1, \ldots, T$. Several problem instances will be generated for different values of the coefficients $\bar{f}, \bar{\kappa}, \bar{c}$. Note that we assume that the capacities are time-invariant to be able to use the valid inequalities presented in the previous section. We assume that all of the nodes defined for the same time period have equal probabilities $\left(\pi_{i}\right)$. These parameter settings are very similar to the ones considered in Guan et al. (2009). Values of the other parameters will be given in the subsequent sections.

We implement the formulations and the branch-and-cut procedure in IBM ILOG Cplex 12.5, and perform the experiments on a $2.4 \mathrm{GHz}$ Intel Core i7 Machine with $16 \mathrm{~GB}$ memory running Windows 10 .

\subsection{Test of valid inequalities}

In this part, we consider the problem under the first setting (S1), the basic form of our formulation in which there is no nervousness cost, to test the valid inequalities developed in the previous section. Since most of the commercial solvers, such as Cplex, can solve MIP formulations with quadratic objective functions efficiently, we consider the following formulation where the quadratic compression cost function is kept in the objective function.

$$
\begin{aligned}
& \min \sum_{t=1}^{T} f_{t} y_{t}+\sum_{i \in V}\left(c_{i} x_{i}+h_{i} s_{i}\right)+\sum_{i \in V} \kappa_{i} r_{i}^{2} \\
& \text { s.t. }(2)-(5) \\
& x_{i}, s_{i}, r_{i} \geq 0 \\
& y_{t} \in\{0,1\}, z_{t} \geq 0 \\
& i \in V \\
& t=1, \ldots, T
\end{aligned}
$$

We consider the following additional parameter settings: $\bar{\kappa}=1, \quad \lambda_{t(i)}=0.5, \quad \delta_{t(i)}=1.3$ for $i \in V$, and different values of setup costs and capacities with $\bar{f} \in\{1000,2000,3000\}$ and $\bar{c} \in$ $\{150,200,250,300\}$. In order to observe the effect of the inequalities we developed, we first turn off presolve and the automatic cuts of Cplex. 


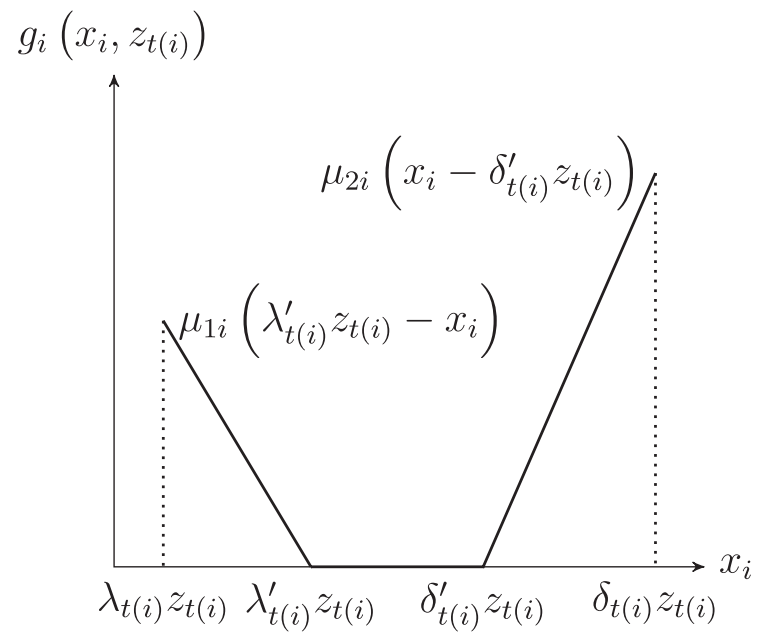

(i)

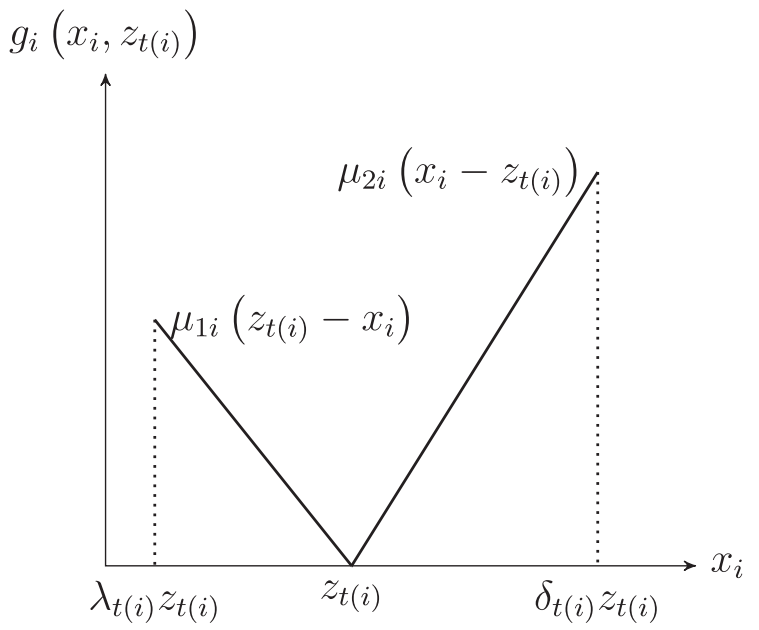

(ii)

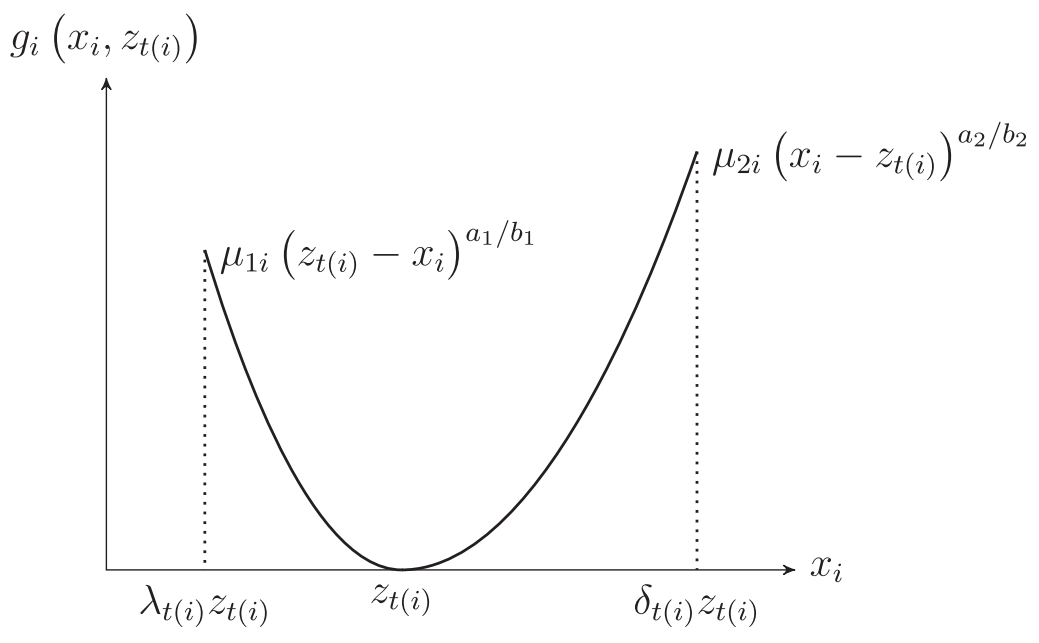

(iii)

Fig. 3. Special cases of the nervousness cost function.

We first test the cycle inequalities developed in Section 5.1. We add the extended formulation (25)-(29) for each node $u \in V$ to the formulation LSQ and solve the resulting formulation by Cplex. Remember that for given $u$, inequalities (25)-(29) are developed for all the descendant nodes of $u, V(u)$. We also consider inequalities (22) for all paths starting at node $u$ and add the extended formulation (25)-(29) for each path $P(u, v)$ for all $v \in V(u)$. Note that an arc is defined between every pair of nodes in $V(u)$ in the graph $G_{u}^{\prime}$ while developing the extended formulation. By considering the extended formulation for each path separately, we exclude the arcs that are defined between the nodes of different paths. We test both of these approaches for small problem instances.

Results for instances with 7 periods can be seen in Table 1 . In this table, the columns rgap, cpu and node represent the root gap (the percentage gap between the best lower bound obtained in the root node and the optimal value), solution time (in seconds) and the number of branch-and-bound nodes explored, respectively. Note that all the problem instances are solved in much smaller time (less than one second) by the formulation LSQ but the main aim of this experiment is to see the improvement of root gap by reformulation. We observe that including the extended formulation reduces the root gap in all of the problem instances. The root gaps are reduced by more than $50 \%$ in all the problem instances. The root gap is decreased from $11.6 \%$ to $3.3 \%$ on the average by both of the reformulations. Although root gaps of the reformulations are very close, adding the extended formulation for each path separately results in less computation time.

We consider the same experiment for larger instances by using only the second approach since it performs better in terms of computation times. Moreover, adding all the inequalities may not be a good approach for solving larger instances. In order to make use of the extended formulation for solving larger instances, we add the extended formulation for paths with maximum $\bar{n}$ nodes. In other words, we add the extended formulation for inequality (22) for the path $P(u, v)$ if $t(v)-t(u) \leq \bar{n}-1$. This approach is very similar to the approximate extended formulations of Van Vyve and Wolsey (2006). Note that, $\bar{n}=T$ corresponds to adding the extended formulation for all paths (the original version of the second approach), and the results for this case give the information about the maximum possible root gap reduction due to the 
Table 1

Test of different extended formulations for $T=7$.

\begin{tabular}{|c|c|c|c|c|c|c|c|c|c|c|}
\hline \multirow[t]{3}{*}{$\bar{f}$} & \multirow[t]{3}{*}{$\bar{c}$} & \multicolumn{3}{|c|}{ LSQ } & \multicolumn{6}{|c|}{ LSQ with reformulation } \\
\hline & & \multirow[t]{2}{*}{ rgap } & \multirow[t]{2}{*}{$\mathrm{cpu}$} & \multirow[t]{2}{*}{ node } & \multicolumn{3}{|c|}{ for $V(u)$} & \multicolumn{3}{|c|}{ for paths } \\
\hline & & & & & rgap & cpu & node & rgap & cpu & node \\
\hline \multirow[t]{4}{*}{1000} & 150 & 3.36 & 0 & 3 & 1.07 & 57 & 1 & 1.08 & 3 & 1 \\
\hline & 200 & 9.05 & 0 & 12 & 2.52 & 88 & 5 & 2.43 & 4 & 6 \\
\hline & 250 & 9.76 & 0 & 10 & 2.08 & 69 & 4 & 2.06 & 4 & 4 \\
\hline & 300 & 12.59 & 0 & 28 & 2.76 & 81 & 7 & 2.95 & 4 & 6 \\
\hline \multirow[t]{4}{*}{2000} & 150 & 5.82 & 0 & 4 & 2.33 & 55 & 4 & 2.33 & 3 & 4 \\
\hline & 200 & 11.73 & 0 & 15 & 3.82 & 72 & 10 & 3.82 & 7 & 10 \\
\hline & 250 & 17.86 & 0 & 17 & 4.59 & 78 & 7 & 4.49 & 14 & 5 \\
\hline & 300 & 19.02 & 0 & 42 & 5.81 & 97 & 12 & 5.84 & 7 & 9 \\
\hline \multirow[t]{4}{*}{3000} & 150 & 9.47 & 0 & 12 & 4.11 & 72 & 8 & 4.12 & 6 & 9 \\
\hline & 200 & 9.89 & 0 & 17 & 3.34 & 69 & 7 & 3.43 & 4 & 4 \\
\hline & 250 & 14.06 & 0 & 35 & 4.47 & 78 & 13 & 4.50 & 21 & 21 \\
\hline & 300 & 16.57 & 0 & 20 & 3.15 & 66 & 4 & 3.14 & 11 & 4 \\
\hline
\end{tabular}

Table 2

Test of the partial reformulation for $T=8$.

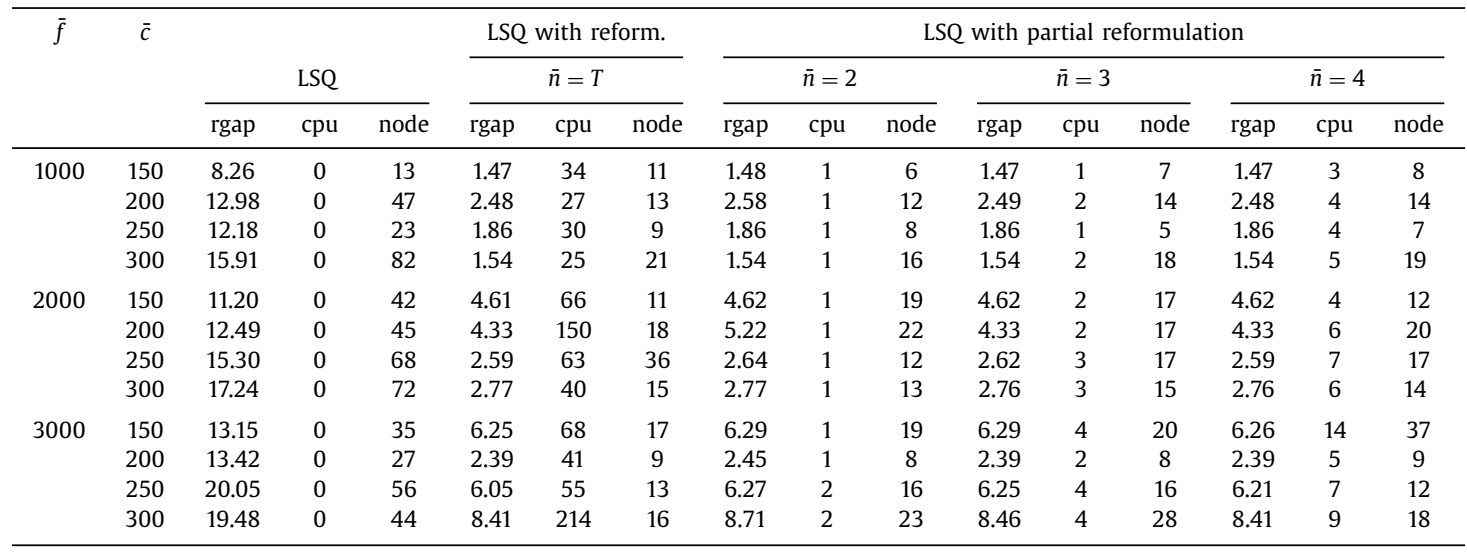

reformulation. We first consider different $\bar{n}$ values in order to see the effect of the reformulation for different $\bar{n}$ values. Results for instances with 8 periods are given in Table 2. LSQ solves these instances again in much smaller time (less than one second), and the smaller $\bar{n}$ values result in less computation times as expected. The root gap is again reduced by more than $50 \%$ by the reformulation (column $\bar{n}=T$ ). Besides, the number of branch and bound nodes explored decreases in all of the problem instances. For example, for one of the instances, adding the extended formulation for all paths including at most two nodes decreases the root gap from $20.05 \%$ to $6.27 \%$.

For solving larger problem instances, we make use of the partial extended formulation and the mixing inequalities (34) and (35). We first add the partial extended formulation for $\bar{n}=2$ for the nodes of the scenario tree defined for the first 6 periods. Then, we solve the relaxation of the reformulation and call the separation routine of mixing inequalities in the root node. The separation routine is called maximum 15 times and at each round at most 100 mixing inequalities are added. We consider 13 and 14 period instances and set the time limit to 3600 seconds.

A summary of our computational experiment for both LSQ and CLSII can be seen in Table 3. After several initial runs, we only report the CLSII with the partial extended formulation and the valid mixing inequalities in further analysis for solving larger problem instances, since they outperform the straightforward CLSII implementation. If the solver terminates with positive optimality gap, the final percentage gap is given under the column (fgap) in parenthesis. The best solution times and the root gaps for each instance are written in bold.
As it can be observed from Table 3, for 13 period instances, the partial reformulation reduces the root gap of LSQ from $19.7 \%$ to $11 \%$ on the average, and the mixing inequalities further reduce this gap about $2 \%$. Similar results are observed for 14 period instances. For these instances, the average root gap of LSQ is reduced from $19.8 \%$ to $12.2 \%$ by the partial reformulation, and average $0.8 \%$ root gap reduction is obtained due to the addition of the valid inequalities. Besides, the root gaps of LSQ and CLSII with the partial extended formulations (and the mixing inequalties) are very close to each other.

The best root gaps are obtained by the addition of the partial extended formulation and the mixing inequalities. But in most of the instances, the best solution times are achieved by the addition of only the partial extended formulation. This might be due to increase in the size of the formulations by the addition of the valid mixing inequalities. Note that the root gap reduction by the mixing inequalities is very small, for example, for the instances with 14 periods, the root gap is decreased by $0.3 \%$ on the average by the addition of the valid inequalities to CLSII with the partial extended formulation. But the addition of these inequalities increases the size of the formulation, and determination of the inequalities to be added (the separation routine) takes some time. These observations are supported by the results for the instances with 14 periods. In most of the instances, the best solution times are due to the addition of the partial extended formulation to either LSQ or CLSII.

Note that, the average solution times of LSQ with the partial extended formulation and CLSII with the partial extended formulation are very close. Besides, in most of the instances, one of them 
Table 3

Results for larger instances.

\begin{tabular}{|c|c|c|c|c|c|c|c|c|c|c|c|c|c|c|c|c|c|}
\hline \multirow[t]{2}{*}{$T$} & \multirow[t]{2}{*}{$\bar{f}$} & \multirow[t]{2}{*}{$\bar{c}$} & \multicolumn{3}{|c|}{ LSQ } & \multicolumn{3}{|c|}{ LSQ reform. } & \multicolumn{3}{|c|}{ LSQ reform. \& cuts } & \multicolumn{3}{|c|}{ CLSII reform. } & \multicolumn{3}{|c|}{ CLSII reform. \& cuts } \\
\hline & & & rgap & cpu (fgap) & node & rgap & cpu (fgap) & node & rgap & cpu (fgap) & node & rgap & cpu (fgap) & node & rgap & cpu (fgap) & node \\
\hline \multirow[t]{13}{*}{13} & \multirow[t]{4}{*}{1000} & 150 & 13.35 & 526 & 627 & 7.61 & 248 & 313 & 7.49 & 224 & 250 & 7.48 & 313 & 200 & 7.38 & 280 & 168 \\
\hline & & 200 & 16.71 & 415 & 593 & 9.44 & 228 & 207 & 8.22 & 212 & 163 & 9.34 & 287 & 238 & 8.96 & 296 & 211 \\
\hline & & 250 & 16.36 & 394 & 628 & 9.07 & 204 & 184 & 6.38 & 161 & 68 & 8.99 & 227 & 200 & 8.49 & 281 & 148 \\
\hline & & 300 & 19.40 & 630 & 928 & 8.32 & 409 & 208 & 5.79 & 415 & 150 & 10.10 & 255 & 148 & 9.35 & 268 & 113 \\
\hline & \multirow[t]{4}{*}{2000} & 150 & 16.07 & 363 & 289 & 8.61 & 140 & 117 & 8.52 & 142 & 114 & 8.15 & 300 & 109 & 8.06 & 285 & 103 \\
\hline & & 200 & 17.01 & 505 & 412 & 10.57 & 230 & 175 & 8.98 & 238 & 144 & 10.06 & 264 & 87 & 9.67 & 301 & 118 \\
\hline & & 250 & 22.76 & 477 & 621 & 11.10 & 248 & 76 & 7.91 & 180 & 90 & 10.70 & 302 & 164 & 10.15 & 294 & 118 \\
\hline & & 300 & 24.93 & 761 & 972 & 13.82 & 640 & 258 & 9.56 & 628 & 223 & 12.93 & 308 & 160 & 12.28 & 386 & 178 \\
\hline & \multirow[t]{4}{*}{3000} & 150 & 17.60 & 243 & 218 & 10.67 & 249 & 164 & 9.71 & 258 & 228 & 9.77 & 325 & 115 & 9.19 & 307 & 105 \\
\hline & & 200 & 20.37 & 296 & 294 & 12.89 & 241 & 172 & 11.09 & 248 & 154 & 11.94 & 318 & 128 & 11.48 & 378 & 132 \\
\hline & & 250 & 22.54 & 302 & 451 & 13.78 & 248 & 206 & 10.89 & 267 & 158 & 12.92 & 292 & 123 & 12.14 & 380 & 138 \\
\hline & & 300 & 29.34 & 728 & 747 & 16.98 & 238 & 150 & 13.19 & 283 & 166 & 14.88 & 271 & 143 & 13.96 & 438 & 144 \\
\hline & \multicolumn{2}{|c|}{ Average } & 19.70 & 470 & 565 & 11.07 & 277 & 186 & 8.98 & 271 & 159 & 10.61 & 288 & 151 & 10.09 & 324 & 140 \\
\hline \multirow[t]{13}{*}{14} & \multirow[t]{4}{*}{1000} & 150 & 12.60 & 1656 & 588 & 7.60 & 882 & 206 & 7.51 & 879 & 222 & 7.50 & 1042 & 230 & 7.42 & 1146 & 192 \\
\hline & & 200 & 16.83 & (3.77) & 900 & 10.41 & 1385 & 312 & 9.95 & 1445 & 283 & 10.21 & 1136 & 217 & 9.98 & 1495 & 227 \\
\hline & & 250 & 19.39 & 2578 & 1268 & 11.71 & 1356 & 362 & 11.16 & 1572 & 384 & 11.59 & 1149 & 291 & 11.28 & 1535 & 309 \\
\hline & & 300 & 18.58 & $(2.48)$ & 1125 & 11.42 & 1225 & 418 & 10.41 & 948 & 281 & 11.37 & 932 & 318 & 10.99 & 1580 & 394 \\
\hline & \multirow[t]{4}{*}{2000} & 150 & 16.44 & 2066 & 335 & 10.49 & 926 & 220 & 10.20 & 1031 & 189 & 9.50 & 1196 & 137 & 9.34 & 1440 & 132 \\
\hline & & 200 & 18.57 & 3070 & 850 & 11.27 & 1186 & 248 & 10.88 & 1007 & 262 & 10.81 & 1525 & 211 & 10.59 & 1795 & 241 \\
\hline & & 250 & 20.96 & 3156 & 1197 & 13.33 & 3117 & 436 & 12.61 & $(2.78)$ & 363 & 12.94 & 1419 & 286 & 12.61 & 1704 & 223 \\
\hline & & 300 & 23.53 & 2143 & 880 & 13.34 & 775 & 173 & 12.00 & 1093 & 183 & 12.87 & 997 & 157 & 12.42 & 1517 & 120 \\
\hline & \multirow[t]{4}{*}{3000} & 150 & 16.87 & 1016 & 241 & 10.43 & 1329 & 216 & 10.15 & 1393 & 214 & 9.52 & 1009 & 108 & 9.35 & 1320 & 121 \\
\hline & & 200 & 20.47 & 1602 & 308 & 13.11 & 840 & 161 & 12.70 & 972 & 173 & 12.14 & 1323 & 140 & 11.88 & 1487 & 136 \\
\hline & & 250 & 24.27 & 1859 & 496 & 16.13 & 1231 & 311 & 15.28 & 1349 & 287 & 15.18 & 1286 & 199 & 14.85 & 2009 & 216 \\
\hline & & 300 & 29.50 & 3494 & 1050 & 17.29 & 805 & 194 & 13.88 & 1036 & 173 & 16.22 & 1312 & 157 & 15.73 & 1680 & 197 \\
\hline & \multicolumn{2}{|c|}{ Average } & 19.83 & 2487 & 1201 & 12.21 & 1255 & 271 & 11.39 & 1360 & 521 & 11.65 & 1194 & 204 & 11.37 & 1559 & 209 \\
\hline
\end{tabular}

achieves the best solution times among all the reformulations. But, in general none of them is superior to the others.

Overall, it can be observed that by conic strengthening, reformulating and adding valid inequalities, we could reduce the root gaps and the solution times for our problem instances. Significant improvements are obtained by the partial reformulation (by adding the partial extended formulation).

In all the experiments presented above, Cplex presolve, heuristics and cuts are disabled to observe the effect of the inequalities we developed. Since the default settings of Cplex performs well on most problems, next we test the reformulations with the default Cplex options. When the valid inequalities are made use of in a branch-and-cut procedure, Cplex switches from the dynamic search algorithm (the method used in the default settings) to the conventional branch-and-cut. We first test CLSII with the partial reformulation and valid inequalities with the enabled Cplex presolve and cuts, and the traditional branch-and-cut. We observe that the solution times of CLSII with partial reformulation and valid inequalities get worse than LSQ. Then, as we obtain the major root gap reduction by the partial reformulation, we test the formulations LSQ and CLSII with only the partial reformulation. Note that in the partial reformulation the inequalities are added a priori, and this enables us to make use of the dynamic search algorithm of Cplex. Since CLSII with the partial reformulation is outperformed by LSQ with the partial reformulation, we only present the results for LSQ and LSQ with the partial reformulation in Table 4. As it can be observed from the table, for the instances with 13 periods, although the solution times are very close to each other, in most of the instances LSQ with partial reformulation performs better. The difference becomes more clear in the problem instances with 14 periods. In 9 of the problem instances, LSQ with the partial reformulation solves the problem in less computation times; and in the remaining 3 instances the solution times get worse due to the increase in the size of the problem by the partial reformulation. Besides, both the improvement and the deterioration due
Table 4

Results with default Cplex options.

\begin{tabular}{|c|c|c|c|c|c|c|}
\hline \multirow[t]{2}{*}{$T$} & \multirow[t]{2}{*}{$\bar{f}$} & \multirow[t]{2}{*}{$\bar{c}$} & \multicolumn{2}{|c|}{ LSQ } & \multicolumn{2}{|c|}{ LSQ reform. } \\
\hline & & & $\mathrm{cpu}$ & node & cpu & node \\
\hline \multirow[t]{12}{*}{13} & \multirow[t]{4}{*}{1000} & 150 & 172 & 132 & 169 & 111 \\
\hline & & 200 & 111 & 89 & 139 & 148 \\
\hline & & 250 & 156 & 191 & 147 & 105 \\
\hline & & 300 & 202 & 209 & 192 & 172 \\
\hline & \multirow[t]{4}{*}{2000} & 150 & 188 & 103 & 137 & 100 \\
\hline & & 200 & 289 & 204 & 197 & 125 \\
\hline & & 250 & 215 & 163 & 198 & 122 \\
\hline & & 300 & 319 & 229 & 251 & 153 \\
\hline & \multirow[t]{4}{*}{3000} & 150 & 259 & 161 & 275 & 151 \\
\hline & & 200 & 249 & 160 & 265 & 146 \\
\hline & & 250 & 386 & 228 & 377 & 228 \\
\hline & & 300 & 417 & 283 & 340 & 204 \\
\hline \multirow[t]{12}{*}{14} & \multirow[t]{4}{*}{1000} & 150 & 485 & 91 & 500 & 89 \\
\hline & & 200 & 772 & 254 & 633 & 234 \\
\hline & & 250 & 660 & 163 & 623 & 199 \\
\hline & & 300 & 1032 & 321 & 868 & 244 \\
\hline & \multirow[t]{4}{*}{2000} & 150 & 843 & 179 & 579 & 103 \\
\hline & & 200 & 895 & 207 & 794 & 122 \\
\hline & & 250 & 1269 & 228 & 961 & 192 \\
\hline & & 300 & 822 & 181 & 498 & 42 \\
\hline & \multirow[t]{4}{*}{3000} & 150 & 914 & 213 & 911 & 159 \\
\hline & & 200 & 932 & 119 & 1094 & 173 \\
\hline & & 250 & 1481 & 242 & 1296 & 171 \\
\hline & & 300 & 1216 & 249 & 1585 & 212 \\
\hline
\end{tabular}

to the partial reformulation is about 190 seconds on the average. We note that when the setup costs are medium sized (2000 in our instances), the partial reformulation improves the solution times more. So we conclude that although Cplex with the default options performs well, its performance highly depends on the problem instance, and it is possible to improve it by using our partial reformulation. 
Table 5

Classical Scenario Tree Formulation (STF) vs. Setting 3 (S3).

\begin{tabular}{ccccccc}
\hline $\bar{f}$ & $\bar{c}$ & \multicolumn{2}{c}{ STF } & S3 & $\begin{array}{c}\text { cost } \\
\text { diff }\end{array}$ & $\begin{array}{c}\% \text { cost } \\
\text { diff }\end{array}$ \\
\cline { 3 - 6 } & & BUB & fgap(\%) & obj & 13,436 & 6.10 \\
\hline \multirow{2}{*}{1000} & 100 & 220,083 & 1.37 & 233,519 & 13,437 & 5.25 \\
& 150 & 218,568 & 1.25 & 230,045 & 11,478 & 1.22 \\
& 200 & 223,425 & 1.02 & 226,140 & 2715 & 8.72 \\
\multirow{2}{*}{3000} & 100 & 266,369 & 3.22 & 289,606 & 23,237 & 5.64 \\
& 150 & 272,528 & 4.42 & 287,899 & 15,371 & 4.71 \\
& 200 & 268,240 & 5.29 & 280,865 & 12,625 & 6.56 \\
& 100 & 304,041 & 6.33 & 323,985 & 19,944 & 3.97 \\
& 150 & 309,659 & 8.39 & 321,950 & 12,291 & 4.98 \\
\hline
\end{tabular}

\subsection{Nervousness}

Now, we will analyze the nervousness of the solutions obtained by our formulation under different settings. Our main aim is to observe the effect of range constraints, nervousness cost function and controllable processing times on the nervousness of the production plans.

In this experiment, we consider problem instances with 13 periods with the nervousness cost function depicted in Fig. 2 where $\lambda^{\prime}=0.5 \lambda$ and $\delta^{\prime}=0.5 \delta$. Additionally, we consider the following parameter settings: the coefficients related to the compression cost $\bar{\kappa} \in\{1,5\}$; the nervousness cost $\mu_{1 i}=\mu_{2 i} \sim \pi_{i} U[0.4 \bar{\mu}, 0.6 \bar{\mu}]$, where $\bar{\mu} \in\{1,5\}$; the setup cost $\bar{f} \in\{1000,2000,3000\}$; and the capacity $\bar{c} \in\{100,150,200\}$. We consider different range parameters: $\lambda_{t}=1-\epsilon, \delta_{t}=1+\epsilon$, where $\epsilon \in\{0.2,0.5,0.8\}$ for $t=1, \ldots, T$. We do not report the solution times, and root gaps in this section, since our main aim is to analyze the nervousness of different solutions. Moreover, in the tables we report the averages of the results for the given parameter settings.

As it is stated in the previous sections, the classical scenario tree formulation of the stochastic lot sizing problem causes both setup and quantity oriented nervousness (we will call that formulation STF), and in our formulation, we exclude setup oriented nervousness. Thus, by comparing the total cost of the solutions of the classical scenario tree formulation and our formulation under the third setting (static-dynamic uncertainty strategy), we can observe the cost of eliminating setup oriented nervousness. This comparison can be seen in Table 5 .

In the classical scenario tree formulation $0-1$ setup variables are defined for each node separately. Thus, the number of binary setup variables in STF $\left(n=m^{T}-1\right)$ is much larger than that of our formulation $(T)$. Therefore, the solver cannot solve STF for 13 period instances to optimality in $3600 \mathrm{~s}$. For this formulation, the final gap at the end of the time limit is given under the column fgap in Table 5. The total cost of the best solution found in 3600 seconds is given under the column BUB.

Cost differences and the percentage cost differences between the solutions of these two formulations are reported under the columns cost diff and \% cost diff. In our comparisons, we use the best upper bounds for the formulation STF. It can be seen from Table 5 that the cost increases about 5\% due to restricting setup decisions from scenarios to periods. In other words, for eliminating setup oriented nervousness we increased the total cost by $5 \%$ on the average. According to the solutions of the classical scenario tree formulation, there is a setup decision for almost each period under different scenarios. On the other hand, for our formulation the average number of production periods is about $11,9,7$ for $\bar{c} \in\{100,150,200\}$, respectively. Consequently, we fix the production periods beforehand and obtain production schedules with no setup oriented nervousness in return for an increase in the total cost.
Now we will examine the effect of the range constraints and nervousness cost function on the quantity oriented nervousness. To do this, we compare the solutions for settings S1, S2, S3, and S8. Note that, these settings correspond to the four possible combinations of the presence of range constraints and nervousness cost function. The results of this experiment are given in Tables 6 and 7 , and we compare the total costs and nervousness costs of the production schedules obtained under these settings for $\bar{\mu}=1$ and $\bar{\mu}=5$, respectively. Note that the objective function under settings S1 and S3 does not contain the nervousness cost of the solution, whereas the one under settings S2 and S8 does. Thus, here we compare the total production, inventory holding, and compression costs of the solutions; and we call them conventional costs. Since setting S3 does not include range constraints and nervousness cost function, this is the most relaxed version, and it results in the minimum cost solutions among all the settings. Therefore, in the left hand sides of these tables, we report the percentage increases in the conventional costs under other settings compared to S3.

In the right parts of the tables, we compare the nervousness costs of the production schedules obtained under settings S1, S2, S3, and S8. Since settings S1 and S3 do not contain nervousness cost functions, we compute the nervousness costs of the solutions obtained under these settings by using our nervousness cost function. Moreover, as setting S3 does not contain the promised production amounts as decision variables, we compute the promised production amounts for each period by taking the average of the actual production amounts for the corresponding nodes. Note that setting S8 results in the most stable production schedules among all these settings, since we include both range constraints and nervousness cost function in this setting. Hence, in these tables, we give the nervousness cost of the solutions obtained under setting S8, and report the ratio of the nervousness costs under other settings to the nervousness cost under setting S8. Although the solutions in settings S3 and S1 do not depend on the parameter $\bar{\mu}$, we give the results for these settings in both tables to make the comparisons more clear.

It can be observed from the tables that nervousness costs of the solutions obtained under settings S2 and S8 are almost the same for $\epsilon \in\{0.5,0.8\}$, and the increases in the conventional costs under these setting are also very close. Thus, we can conclude that in the presence of a nervousness cost, the range constraints do not have a significant impact unless they are very tight. But, if the range parameters are closer to one ( $\epsilon=0.2$ in our instances), then including range constraints also decrease the nervousness costs. Moreover, for $\bar{\mu}=1$ (when the nervousness cost coefficients are smaller) in settings S2 and S8, we decrease nervousness costs of the production schedules in larger amounts by increasing the conventional costs. For example, by increasing the conventional costs about $4.3 \%$, the ratio of the nervousness costs is decreased by 337,406 on average under setting S2 for $\epsilon=0.8$.

Note that nervousness costs of the solutions under settings S1 and S3 are very large compared to settings S2 and S8. Since setting S3 does not include range constraints and nervousness cost function, this setting has the largest nervousness cost. In setting S1, since there is no penalty cost for the differences between the promised production amounts and the actual production amounts, the actual production amounts are spread on the interval determined by the range constraints. On the other hand, as in settings S2 and S8 the differences between the production amounts and the promised production amounts are penalized, the actual production amounts become very close to the promised production amounts. This is the reason for the large differences in the nervousness costs of these settings.

By comparing nervousness costs of the solutions obtained under settings S1 and S3, we can conclude that including only range constraints also decreases the nervousness costs of the 
Table 6

Comparison of nervousness and conventional costs for $\overline{\boldsymbol{\mu}}=\mathbf{1}$.

\begin{tabular}{|c|c|c|c|c|c|c|c|c|c|c|}
\hline$\overline{\mathbf{f}}$ & $\overline{\mathbf{c}}$ & S3 obj & $\epsilon$ & \multicolumn{3}{|c|}{$\begin{array}{l}\text { \% increase in the } \\
\text { conventional costs }\end{array}$} & \multicolumn{4}{|c|}{ Nervousness costs } \\
\hline \multirow[t]{7}{*}{1000} & \multirow[t]{3}{*}{100} & \multirow[t]{3}{*}{233,519} & 0.8 & 0.39 & 5.28 & 5.28 & 295 & 1.00 & 203,330 & 360,418 \\
\hline & & & 0.5 & 3.02 & 11.75 & 11.75 & 1457 & 1.01 & 32,642 & 128,145 \\
\hline & & & 0.2 & 18.76 & 25.68 & 28.99 & 3806 & 1.88 & 3881 & 79,035 \\
\hline & 150 & 230,024 & 0.2 & 26.77 & 37.18 & 43.91 & 5522 & 1.93 & 4286 & 56,390 \\
\hline & \multirow[t]{3}{*}{200} & \multirow[t]{3}{*}{226,140} & 0.8 & 0.49 & 5.72 & 5.72 & 295 & 1.00 & 206,698 & 417,450 \\
\hline & & & 0.5 & 3.24 & 14.54 & 14.58 & 1846 & 1.00 & 25,807 & 106,973 \\
\hline & & & 0.2 & 30.79 & 42.04 & 48.76 & 5333 & 2.03 & 4009 & 56,503 \\
\hline \multirow[t]{5}{*}{2000} & 100 & 288,883 & 0.8 & 0.35 & 3.65 & 3.65 & 180 & 1.00 & 349,033 & 583,342 \\
\hline & 150 & 287,452 & 0.2 & 19.19 & 29.81 & 34.40 & 6020 & 1.76 & 3911 & 38,899 \\
\hline & \multirow[t]{3}{*}{200} & \multirow[t]{3}{*}{280,865} & 0.8 & 0.19 & 3.76 & 3.76 & 174 & 1.00 & 170,831 & 234,584 \\
\hline & & & 0.5 & 1.87 & 10.74 & 10.74 & 1499 & 1.01 & 25,618 & 59,266 \\
\hline & & & 0.2 & 19.10 & 29.74 & 34.64 & 5500 & 1.88 & 4219 & 31,317 \\
\hline \multirow[t]{7}{*}{3000} & \multirow[t]{3}{*}{100} & \multirow[t]{3}{*}{322,853} & 0.8 & 0.22 & 2.50 & 2.50 & 246 & 1.00 & 190,469 & 310,476 \\
\hline & & & 0.5 & 1.43 & 6.14 & 6.14 & 1210 & 1.00 & 27,422 & 109,926 \\
\hline & & & 0.2 & 10.56 & 15.78 & 17.38 & 3700 & 1.64 & 4217 & 59,692 \\
\hline & \multirow[t]{3}{*}{150} & \multirow[t]{3}{*}{321,573} & 0.8 & 0.14 & 3.19 & 3.19 & 220 & 1.00 & 136,676 & 173,627 \\
\hline & & & 0.5 & 1.48 & 8.99 & 8.99 & 1468 & 1.00 & 26,098 & 58,146 \\
\hline & & & 0.2 & 16.48 & 25.69 & 29.83 & 5765 & 1.81 & 4059 & 29,097 \\
\hline & 200 & 316,472 & 0.8 & 0.20 & 3.41 & 3.41 & 265 & 1.00 & 142,963 & 188,852 \\
\hline
\end{tabular}

Table 7

Comparison of nervousness and conventional costs for $\overline{\boldsymbol{\mu}}=\mathbf{5}$.

\begin{tabular}{|c|c|c|c|c|c|c|c|c|c|c|}
\hline \multirow[t]{2}{*}{$\bar{f}$} & \multirow[t]{2}{*}{$\bar{c}$} & \multirow[t]{2}{*}{ S3 obj } & \multirow[t]{2}{*}{$\epsilon$} & \multicolumn{3}{|c|}{$\begin{array}{l}\text { \% increase in the } \\
\text { conventional costs }\end{array}$} & \multicolumn{4}{|c|}{ Nervousness costs } \\
\hline & & & & S1 & $\mathrm{S} 2$ & S8 & S8 & S2 & S1 & S3 \\
\hline \multirow[t]{7}{*}{1000} & \multirow[t]{3}{*}{100} & \multirow[t]{3}{*}{233,519} & 0.8 & 0.39 & 5.48 & 5.48 & 65 & 1.00 & $4,645,414$ & $8,234,351$ \\
\hline & & & 0.5 & 3.02 & 12.86 & 12.86 & 649 & 1.00 & 366,322 & $1,438,119$ \\
\hline & & & 0.2 & 18.76 & 31.20 & 31.68 & 2990 & 1.25 & 4940 & 503,091 \\
\hline & 150 & 230,024 & 0.2 & 26.77 & 44.99 & 48.08 & 3589 & 1.85 & 6595 & 433,829 \\
\hline & \multirow[t]{3}{*}{200} & \multirow[t]{3}{*}{226,140} & 0.8 & 0.49 & 5.93 & 5.93 & 65 & 1.00 & $4,722,168$ & $9,536,953$ \\
\hline & & & 0.5 & 3.24 & 15.84 & 15.89 & 856 & 1.08 & 278,121 & $1,152,831$ \\
\hline & & & 0.2 & 30.79 & 49.85 & 52.85 & 4121 & 1.58 & 5187 & 365,579 \\
\hline \multirow[t]{5}{*}{2000} & 100 & 288,883 & 0.8 & 0.35 & 3.75 & 3.75 & 54 & 1.00 & $5,816,689$ & $9,721,494$ \\
\hline & 150 & 287,452 & 0.2 & 19.19 & 36.11 & 37.92 & 3345 & 1.77 & 7038 & 350,007 \\
\hline & \multirow[t]{3}{*}{200} & \multirow[t]{3}{*}{280,865} & 0.8 & 0.19 & 3.86 & 3.86 & 36 & 1.00 & $4,087,856$ & $5,613,396$ \\
\hline & & & 0.5 & 1.87 & 11.58 & 11.58 & 761 & 1.00 & 252,246 & 583,549 \\
\hline & & & 0.2 & 19.10 & 35.92 & 37.68 & 3322 & 1.74 & 6985 & 259,254 \\
\hline \multirow[t]{7}{*}{3000} & \multirow[t]{3}{*}{100} & \multirow[t]{3}{*}{322,853} & 0.8 & 0.22 & 2.62 & 2.62 & 118 & 1.00 & $1,986,055$ & $3,237,398$ \\
\hline & & & 0.5 & 1.43 & 6.77 & 6.77 & 754 & 1.00 & 219,892 & 881,487 \\
\hline & & & 0.2 & 10.56 & 18.97 & 19.26 & 2902 & 1.23 & 5375 & 380,435 \\
\hline & \multirow[t]{3}{*}{150} & \multirow[t]{3}{*}{321,573} & 0.8 & 0.14 & 3.30 & 3.30 & 47 & 1.00 & $3,199,185$ & $4,064,083$ \\
\hline & & & 0.5 & 1.48 & 9.73 & 9.73 & 755 & 1.00 & 253,691 & 565,213 \\
\hline & & & 0.2 & 16.48 & 31.35 & 32.85 & 3395 & 1.71 & 6893 & 247,058 \\
\hline & 200 & 316,472 & 0.8 & 0.20 & 3.55 & 3.55 & 75 & 1.00 & $2,527,866$ & $3,339,269$ \\
\hline
\end{tabular}


solutions. Moreover, including tighter range constraints $(\epsilon=0.2)$ causes larger cost increases but results in more stable production schedules. The cost increase due to range constraints is about $0.3 \%$, $2.3 \%$, and $19 \%$ for $\epsilon=0.8, \epsilon=0.5$, and $\epsilon=0.2$ respectively.

We can observe the effect of nervousness cost coefficient by comparing Tables 6 and 7. Note that as nervousness cost coefficient increases the conventional cost increases slightly; but the nervousness cost decreases in larger amounts. Thus, larger nervousness cost coefficients result in solutions with less quantity oriented nervousness. This is due to the structure of the nervousness cost function; since we assume that the function is convex, we restrict larger deviations from the desired ranges. Furthermore, we have observed that compressing the processing times is mostly used when setup costs are larger and capacities are medium sized with respect to the demand. Moreover, in most of the instances controlling the processing times results in solutions with less nervousness costs.

In summary, our computational results clearly indicate that we could significantly reduce the nervousness costs and generate stable plans with a relatively small increase in conventional costs. Nonlinear nervousness and compression cost functions provide a more realistic production environment, but complicate the problem significantly. We have developed new valid inequalities and utilize the recent advances in second-order cone programming to alleviate this difficulty.

Determining the production periods and the quantities beforehand may ease the other planning issues in a plant such as replenishment of raw materials, inventory control, workforce planning, and agreements with the customers. Based on our computational experiments, we conclude that with a small increase in the total cost ( $7 \%$ in our experiments) one can determine the production periods beforehand. Moreover, since the promised production amounts are also decided before the demand realizations, they will be useful in these arrangements as well. The differences between the promised production amounts and the actual production amounts can be reduced by means of the range constraints and the nervousness cost functions. Of course, the flexibility of the system is very important to determine the values of nervousness related parameters. By a convex nervousness cost function, the difference can be minimized, but it may result in higher costs. On the other hand, even with loose range parameters it is possible to keep the actual production amounts within a range of the promised production amounts with a slight increase in the total cost.

\section{Conclusions}

In this paper, we study the multistage stochastic lot sizing problem with controllable processing times under nervousness considerations. In order to reduce the system nervousness caused by multistage stochastic programming, we restrict the production decisions under different scenarios. To the best of our knowledge, this problem is not studied before. We derive valid inequalities and extended formulation based on continuous mixing and mixing set relaxations of the problem. We computationally test these and observe that the valid inequalities reduce the root gaps significantly and the extended formulations improve the computation times.

In our computational experiments, we also investigate the effect of range constraints and nervousness cost function on the quantity oriented nervousness of the solutions. We observe that including the range constraints and/or the nervousness cost function result in more stable production schedules. Moreover, if there exist a nervousness cost function, then the inclusion of range constraints has a slight impact on the quantity oriented nervousness. Clearly, the results we obtain depend highly on the structure of the nervousness cost function. It may be interesting to investigate different forms of this function and the impact on the solutions.
To limit nervousness, we imposed additional constraints on the recourse variables in a multistage stochastic programming setting. The same approach may be used for other problems where large changes in the recourse decisions have negative consequences in the system performance.

We penalized the difference between the actual production amounts and the intervals determined according to the promised production amounts to reduce quantity oriented nervousness. Considering chance constraints to ensure that the range constraints are satisfied with some certain probability might be another interesting future research direction.

By taking the production decisions for periods, independently from demand realizations, we exclude the setup oriented nervousness from our model. Moreover, this assumption reduces the number of binary decision variables in our formulations and enables us to solve larger problem instances compared to the classical scenario tree approach. However, the proposed solution approach is still limited by the size of the scenario tree, and it may not be possible to solve problem instances with longer planning horizons. On the other hand, as it is also harder to determine the possible demand realizations for further periods, this method can be applied in two different ways. The first is to implement the method in a rolling horizon framework: when the new demand information is obtained, the formulation can be solved with the new data and the updated inventory amounts. This implementation may cause nervousness as the previous production decisions may be changed in the rolling horizon framework, but one can freeze the previous decisions to overcome this drawback. Another possibility is to use the demand estimations for further periods instead of possible demand scenarios. In other words, one can construct a scenario tree in which the possible demand realizations are considered until a period, say period $T^{\prime}$, and after $T^{\prime}$ each node has only one child that represents the estimated demand. In this way, both difficulties may be surpassed: the demand information for further periods can be taken into consideration, and the problem for longer planning horizons can be solved as well.

\section{Acknowledgments}

The authors thank the associate editor and two anonymous referees for their constructive comments and suggestions that significantly improved this paper. This research is supported by TUBITAK Project no $112 \mathrm{M} 220$.

\section{References}

Ahmed, S., King, A.J., Parija, G., 2003. A multi-stage stochastic integer programming approach for capacity expansion under uncertainty. J. Global Optim. 26 (1), 3-24.

Ahuja, R.K., Magnanti, T.L., Orlin, J.B., 1993. Network Flows: Theory, Algorithms, and Applications. Prentice Hall.

Aktürk, M.S., Atamtürk, A., Gürel, S., 2009. A strong conic quadratic reformulation for machine-job assignment with controllable processing times. Oper. Res. Lett. 37 (3), 187-191.

Atamtürk, A., Hochbaum, D.S., 2001. Capacity acquisition, subcontracting, and lot sizing. Manage. Sci. 47 (8), 1081-1100.

Baker, K.R., 1977. An experimental study of the effectiveness of rolling schedules in production planning. Decis. Sci. 8 (1), 19-27.

Ben-Tal, A., Nemirovski, A., 2001. Lectures on modern convex optimization: analysis, algorithms, and engineering applications. Soc. Industr. Appl. Math.

Blackburn, J.D., Kropp, D.H., Millen, R.A., 1986. A comparison of strategies to dampen nervousness in MRP systems. Manage. Sci. 32 (4), 413-429.

Bookbinder, J.H., Tan, J.-Y., 1988. Strategies for the probabilistic lot-sizing problem with service-level constraints. Manage. Sci. 34 (9), 1096-1108.

Brandimarte, P., 2006. Multi-item capacitated lot-sizing with demand uncertainty. Int. J. Prod. Res. 44 (15), 2997-3022.

Cristobal, M.P., Escudero, L.F., Monge, J.F., 2009. On stochastic dynamic programming for solving large-scale planning problems under uncertainty. Comput. Oper. Res. 36 (8), 2418-2428.

De Kok, T., Inderfurth, K., 1997. Nervousness in inventory management: comparison of basic control rules. Eur. J. Oper. Res. 103 (1), 55-82.

Di Summa, M., Wolsey, L.A., 2008. Lot-sizing on a tree. Oper. Res. Lett. 36 (1), 7-13. 
Escudero, L.F., Kamesam, P., 1995. On solving stochastic production planning problems via scenario modelling. TOP 3 (1), 69-95.

Guan, Y., 2011. Stochastic lot-sizing with backlogging: computational complexity analysis. J. Global Optim. 49 (4), 651-678.

Guan, Y., Ahmed, S., Miller, A.J., Nemhauser, G.L., 2006. On formulations of the stochastic uncapacitated lot-sizing problem. Oper. Res. Lett. 34 (3), 241-250.

Guan, Y., Ahmed, S., Nemhauser, G.L., 2009. Cutting planes for multistage stochastic integer programs. Oper. Res. 57 (2), 287-298.

Guan, Y., Ahmed, S., Nemhauser, G.L., Miller, A.J., 2006. A branch-and-cut algorithm for the stochastic uncapacitated lot-sizing problem. Math. Program. 105 (1), 55-84.

Günlük, O., Pochet, Y., 2001. Mixing mixed-integer inequalities. Math. Program. 90 (3), 429-457.

Halman, N., Klabjan, D., Mostagir, M., Orlin, J., Simchi-Levi, D., 2009. A fully polynomial-time approximation scheme for single-item stochastic inventory control with discrete demand. Math. Oper. Res. 34 (3), 674-685.

Heisig, G., 2001. Comparison of (s, S) and (s, nQ) inventory control rules with respect to planning stability. Int. J. Prod. Econ. 73 (1), 59-82.

Helber, S., Sahling, F., Schimmelpfeng, K., 2013. Dynamic capacitated lot sizing with random demand and dynamic safety stocks. OR Spectrum 35, 75-105.

Inderfurth, K., 1994. Nervousness in inventory control: analytical results. Oper.-Res.-Spektrum 16 (2), 113-123.

Kadipasaoglu, S., Sridharan, S., 1997. Measurement of instability in multi-level MRP systems. Int. J. Prod. Res. 35 (3), 713-737.

Kazan, O., Nagi, R., Rump, C.M., 2000. New lot-sizing formulations for less nervous production schedules. Comput. Oper. Res. 27 (13), 1325-1345.

Kilic, O.A., Tarim, S.A., 2011. An investigation of setup instability in non-stationary stochastic inventory systems. Int. J. Prod. Econ. 133 (1), 286-292.

Koca, E., Yaman, H., Aktürk, M.S., 2015. Stochastic lot sizing problem with controllable processing times. Omega 53, 1-10.
Kropp, D.H., Carlson, R.C., Jucker, J.V., 1983. Heuristic lot-sizing approaches for dealing with MRP system nervousness. Decis. Sci. 14 (2), 156-169.

Merzifonluoğlu, Y., Geunes, J., Romeijn, H.E., 2007. Integrated capacity, demand, and production planning with subcontracting and overtime options. Nav. Res. Logist. 54 (4), 433-447.

van Norden, L., van de Velde, S., 2005. Multi-product lot-sizing with a transportation capacity reservation contract. Eur. J. Oper. Res. 165 (1), 127-138.

Pochet, Y., Wolsey, L.A., 2006. Production Planning by Mixed Integer Programming. Springer.

Pujawan, I.N., 2004. Schedule nervousness in a manufacturing system: a case study. Product. Plann. Control 15 (5), 515-524.

Scarf, H., 1960. The optimality of (s, S) policies in the dynamic inventory problem. Math. Methods Soc. Sci.

Shabtay, D., Steiner, G., 2007. A survey of scheduling with controllable processing times. Discrete Appl. Math. 155 (13), 1643-1666.

Simpson, N., 2001. Questioning the relative virtues of dynamic lot sizing rules. Comput. Oper. Res. 28 (9), 899-914.

Tang, L., Che, P., Liu, J., 2012. A stochastic production planning problem with nonlinear cost. Comput. Oper. Res. 39 (9), 1977-1987.

Tunc, H., Kilic, O.A., Tarim, S.A., Eksioglu, B., 2013. A simple approach for assessing the cost of system nervousness. Int. J. Prod. Econ. 141 (2), 619-625.

Tunc, H., Kilic, O.A., Tarim, S.A., Eksioglu, B., 2016. The stochastic lot sizing problem with piecewise linear concave ordering costs. Comput. Oper. Res. 65, 104-110.

Van Vyve, M., 2005. The continuous mixing polyhedron. Math. Oper. Res. 30 (2), 441-452.

Van Vyve, M., Wolsey, L.A., 2006. Approximate extended formulations. Math. Program. 105 (2-3), 501-522.

Vladimirou, H., Zenios, S.A., 1997. Stochastic linear programs with restricted recourse. Eur. J. Oper. Res. 101 (1), 177-192.

Wagner, H.M., Whitin, T.M., 1958. Dynamic version of the economic lot size model. Manage. Sci. 5 (1), 89-96. 